\title{
adPerf: Characterizing the Performance of Third-party Ads
}

\author{
BEHNAM POURGHASSEMI, University of California, Irvine, USA \\ JORDAN BONECUTTER, University of California, Irvine, USA \\ ZHOU LI, University of California, Irvine, USA \\ APARNA CHANDRAMOWLISHWARAN, University of California, Irvine, USA
}

\begin{abstract}
Monetizing websites and web apps through online advertising is widespread in the web ecosystem, creating a billion-dollar market. This has led to the emergence of a vast network of tertiary ad providers and ad syndication to facilitate this growing market. Nowadays, the online advertising ecosystem forces publishers to integrate ads from these third-party domains. On the one hand, this raises several privacy and security concerns that are actively being studied in recent years. On the other hand, the ability of today's browsers to load dynamic web pages with complex animations and Javascript has also transformed online advertising. This can have a significant impact on webpage performance. The latter is a critical metric for optimization since it ultimately impacts user satisfaction. Unfortunately, there are limited literature studies on understanding the performance impacts of online advertising which we argue is as important as privacy and security.

In this paper, we apply an in-depth and first-of-a-kind performance evaluation of web ads. Unlike prior efforts that rely primarily on adblockers, we perform a fine-grained analysis on the web browser's page loading process to demystify the performance cost of web ads. We aim to characterize the cost by every component of an ad, so the publisher, ad syndicate, and advertiser can improve the ad's performance with detailed guidance. For this purpose, we develop a tool, adPerf, for the Chrome browser that classifies page loading workloads into ad-related and main-content at the granularity of browser activities. Our evaluations show that online advertising entails more than $15 \%$ of browser page loading workload and approximately $88 \%$ of that is spent on JavaScript. On smartphones, this additional cost of ads is $7 \%$ lower since mobile pages include fewer and well-optimized ads. We also track the sources and delivery chain of web ads and analyze performance considering the origin of the ad contents. We observe that 2 of the well-known third-party ad domains contribute to $35 \%$ of the ads performance cost and surprisingly, top news websites implicitly include unknown third-party ads which in some cases build up to more than $37 \%$ of the ads performance cost.
\end{abstract}

\section{CCS Concepts: • Software and its engineering $\rightarrow$ Software design engineering; $\bullet$ Information systems $\rightarrow$ Display advertising; • Networks $\rightarrow$ Network performance analysis.}

Additional Key Words and Phrases: Third-party online ads; page load time; fine-grained performance measurement and analysis; Chrome browser

\section{ACM Reference Format:}

Behnam Pourghassemi, Jordan Bonecutter, Zhou Li, and Aparna Chandramowlishwaran. 2021. adPerf: Characterizing the Performance of Third-party Ads. Proc. ACM Meas. Anal. Comput. Syst. 5, 1, Article 3 (March 2021), 26 pages. https://doi.org/10.1145/3447381

Authors' addresses: Behnam Pourghassemi, University of California, Irvine, Irvine, CA, USA, bpourgha@uci.edu; Jordan Bonecutter, University of California, Irvine, Irvine, CA, USA, jbonecut@uci.edu; Zhou Li, University of California, Irvine, Irvine, CA, USA, zhoul15@uci.edu; Aparna Chandramowlishwaran, University of California, Irvine, Irvine, CA, USA, amowli@uci.edu.

Permission to make digital or hard copies of part or all of this work for personal or classroom use is granted without fee provided that copies are not made or distributed for profit or commercial advantage and that copies bear this notice and the full citation on the first page. Copyrights for third-party components of this work must be honored. For all other uses, contact the owner/author(s).

(C) 2021 Copyright held by the owner/author(s).

2476-1249/2021/3-ART3

https://doi.org/10.1145/3447381

Proc. ACM Meas. Anal. Comput. Syst., Vol. 5, No. 1, Article 3. Publication date: March 2021. 


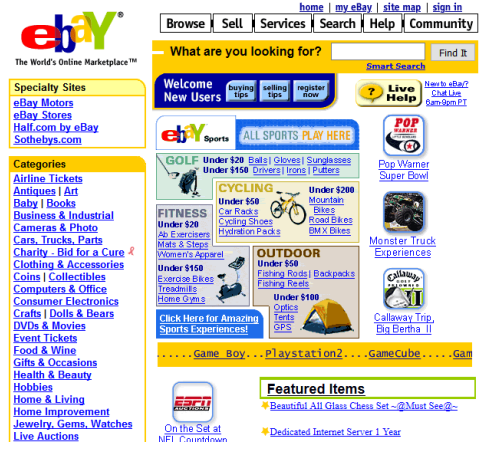

(a) ebay. com in 2002

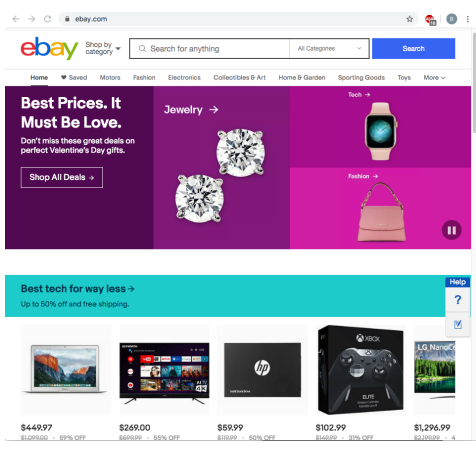

(b) ebay.com in 2020

Fig. 1. Evolution of ads on the web. (a) Early web ads contain text, image, and hyperlink. (b) Today's complex and dynamic web ads (rotating on top of the website) contain JavaScript, animation, multimedia, and iframe.

\section{INTRODUCTION}

Online advertising (essentially display ads on websites) has proliferated in the last decade to the extent where it is now an integral part of the web ecosystem with a multi-billion dollar market $[14,33,35]$. Today, publishers display multiple advertisements (or ads) through pop-ups, banners, click-throughs, iframes, widgets, etc., to monetize their websites and web apps. The majority of these ads neither come from the publisher (website) nor a specific domain. They are delivered through a chain of third-party content providers (such as ad providers, syndication agencies, ad exchange traders, trackers, and analytics service providers) who are part of a complex ad network on the server-side [39]. The current ad delivery method forces publishers to embed unknown third-party content (such as JavaScript or HTML) on their website which could jeopardize user privacy and security. There have been several studies in recent years to locate the untrusted sources and malicious ad contents [27, 28, 34, 39, 42, 43]. Accordingly, different blocking and evasion policies have been devised to guard against such malware and aggressive tracking $[28,41,57,58]$. While user privacy and security are of paramount importance, it is not the solitary concern of the worldwide web community. Online advertising also has a direct impact on website performance (eg., page load time) and in turn user satisfaction. According to Google, 53\% of mobile site visitors leave a page that takes longer than three seconds to load [26].

Web ads have become more diverse and complex keeping up with the pace of advances in web design. Figure 1 compares advertising on ebay.com in 2002 and 2020. As we can observe, in the past, ads only included hypertext and images. However, today's online ads comprise of JavaScript, iframe, animation, multimedia, etc. Evaluating and displaying these dynamic ad contents demand increased computation from the browser and competition for the user's device resources. Coupling this observation with recent studies $[44,48,54]$ that show that most of the page load time is spent on computation activities in modern browsers raise three key questions:

- How much do ads increase the browser's page loading workload?

- What type of web documents and browser activities contribute most to this workload?

- What kind of sources deliver web-ads and how much do they contribute to its performance cost?

Gaining insight into the above questions and understanding how much ads contribute to the breakdown of different activities in modern browsers can inform the design of efficient ads and optimizations targeting those specific activities. Unfortunately, only a handful of studies [30, 36, 38, 
50] have been devoted to the performance analysis of ads, yet many such important open questions remain to be answered.

Previous studies revolving around the performance analysis of ads lack a comprehensive examination for at least the following reasons. First, the majority of them concentrate on the network data traffic overhead, neglecting the performance cost of browser computation activities such as rendering activities $[36,50]$. Second, prior efforts fundamentally share the same approach for quantifying the performance of ads. They use ad-blockers to block websites' ad contents and assess the performance overhead via comparison with vanilla run (no ad-blocking). This approach, however, is prone to inaccuracy as it does not take into account the intrinsic overhead of the ad-blocker. Our measurement of over 350 websites shows that Adblock Plus [4]-the most popular ad-blocker-adds $32 \%$ overhead (median) to the page loading even though it reduces the overall page load time by aggressive content blocking. Furthermore, ad-blockers lead to site breakage and undesired app functionality, particularly, with the prevalence of anti-ad-blockers [38, 40]. Finally, the ad-blocker approach suffers from an inability to perform comprehensive and fined-grained performance analysis. This stems from the way ad-blockers operate where ad-related content is blocked as early as the initiation of network requests. Thus, subsequent ad-related activities such as content parsing, descendent resource loading, and rendering remain invisible for inspection.

In this paper, we investigate the performance overhead of all types of ad-related content by crawling over 500 websites on different systems (laptop and smartphone). Unlike previous efforts, we take a novel approach based on in-browser profiling that does not rely on ad-blockers. Our methodology allows the browser to automatically fetch and evaluate ads' performance at scale. It correlates the browser's computation and network activities to the associated ad contents and quantifies the added cost of ads. We break down the performance overhead to individual requests and content types through a resource mapping technique. This procedure contrives a more robust and detailed performance analysis. Moreover, we demystify and track down ad components on the publisher and characterize the performance overhead considering the origin of ads and how they are delivered to the publisher. To the best of our knowledge, this is the first time such an experiment has been conducted.

Contributions and Findings. To summarize, this paper makes the following contributions.

- We employ a different yet more appropriate methodology to characterize the performance overhead of ads. Our method avoids using ad-blockers, yielding higher accuracy and capability for fine-grained measurements while suppressing site breakages and app failures observed in prior studies. We develop a tool, adPerf based on our technique for the Chrome browser since it is the most commonly used browser by desktop and mobile users. The key challenge we encountered is how to align the performance cost with individual components within an ad (e.g., image and JavaScript code), and we address this through a carefully designed resource mapper (section 3).

- Using adPerf, we perform an in-depth and comprehensive evaluation to demystify and locate the performance cost of web ads. We crawl and analyze over 500 websites from different categories. Our large-scale examination leads to several first-of-a-kind findings that shed light on the performance cost of ads, giving website builders and web ad providers a deeper understanding to mitigate the performance penalty of ads. For example, our results show that on average $15 \%$ of browser page loading activities are spent on ad-related content for Alexa top 350 news websites (section 6).

- To perform a detailed source-to-target analysis of web ads, we construct the dependency graph for the website's resources and track the delivery chain involved in third-party ads. The results show that googletagservices.com and doubleclick. net, two reputable ad 
domains, contribute $35 \%$ of the ad resources resulting in the largest performance cost of online advertising. Moreover, we characterize the trustworthiness and prevalence of third-party ad domains and distinguish the performance overhead of such domains on the web ecosystem. Almost half of the highly-visited websites implicitly trust uncommon third-party ad domains and our results show that about $37 \%$ of ads performance cost is related to untrusted ad domains (section 6.3).

- To assess the impact of the platform on the performance overhead of ads, we compare our performance evaluations on a laptop connected to high-speed WiFi with a smartphone connected to a cellular network. We observe ads cost $7 \%$ less on a smartphone, both in rendering and network, as they are comparatively less prevalent and highly optimized in mobile pages (section 6.4).

- adPerf and the detailed measurement results are available at https://gitlab.com/adPerf/adPerf. adPerf can be leveraged by web researchers and developers for deeper performance evaluation of ads and reducing the overhead of ads. Specifically, we present two compelling use-cases of adPerf for the efficient design of ad intervention and to improve the performance of ads in section 6.5. Additionally, we compare our takeaways with previous studies in the literature (section 7), and discuss similarities and inconsistencies.

\section{BACKGROUND}

In this section, we first outline the limitations of the current approaches in analyzing the performance of ads. Then, we provide an overview of the browser's internal design and workflow.

\subsection{Ad blocking and performance analysis}

Ad blocking is a defense mechanism against advertising and tracking that is wildly deployed by end-users. According to Statistica [19], the global number of clients with connected devices to ad blockers is steadily increasing, and more than a quarter of Internet users in the US were blocking ads in 2019 [2]. Popular ad blockers such as AdBlock [3], Adblock Plus [4], uBlock [22], and Ghostery [13] install as browser add-ons and use filter lists to block web ads and trackers. While user privacy and security are crucial, even ads that are safe and not tracking users can have a significant performance impact that has cascading effects on user satisfaction and Internet costs. Some notable studies [30, 36, 50, 52, 56] lean on ad blockers to measure the performance cost of web ads. The key distinction between our approach and prior efforts is that we do not rely on ad blockers and content-blocking for performance analysis of ads for three main reasons:

Overhead. Multiple studies [36, 38,53] report ad blockers themselves have significant performance overhead due to exhaustive filter-list matching, tracking services of their own, and running background scripts. Our results also affirm this observation. We analyze AdBlock Plus by creating a modified version that still performs all of the content filtering operations without actually blocking any of the content. We calculate the overhead imposed by these filtering operations by measuring the difference in page load times from the modified version of Adblock Plus to the vanilla instance of Chrome. Figure 2 shows the overhead of Adblock Plus on 350 webpages in our corpus (see section 5). According to the figure, for half of the websites, Adblock Plus adds more than $32 \%$ overhead to the page loading due to excessive and CPU-intensive filter rule matching and add-on background activities. Although it may ultimately reduce page loading workload and network cost by aggressive content blocking, it's an inaccurate tool for studying the performance impact of ads.

Functionality. As ad blockers become a threat to the "free" web business model, many websites prevent displaying their content to the visitors that use ad blockers. In this case, the publisher includes a script such as IAB ad block detection script [1] that monitors the visibility of ads to DEAL (Detect, Explain, Ask, Limit) with ad blockers [45]. Typically, when the publisher detects a hidden

Proc. ACM Meas. Anal. Comput. Syst., Vol. 5, No. 1, Article 3. Publication date: March 2021. 


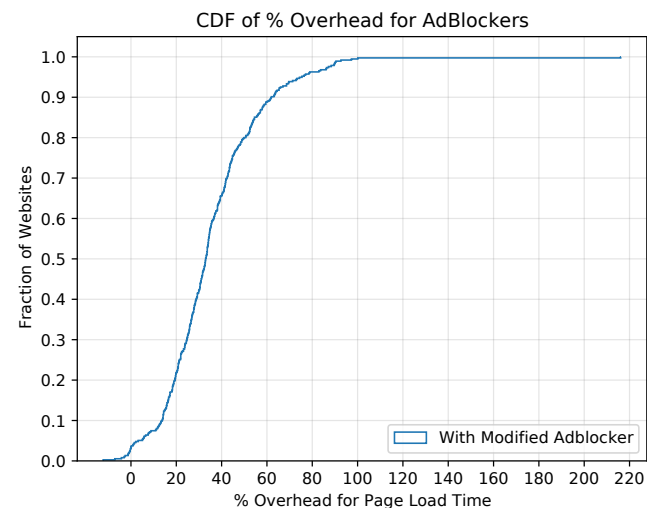

Fig. 2. CDF distribution of AdblockPlus overhead on the page loading of 350 webpages.

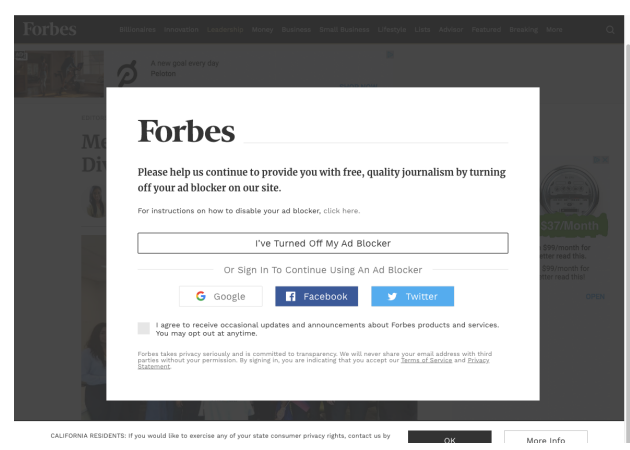

Fig. 3. Snapshot of www. forbes . com. This website prevents loading contents if visitors attempt to block ads.

or removed ad, it immediately stops loading the website by displaying a popup that asks the visitor to turn off the ad-blocker. Figure 3 shows a snapshot of the content-blocking of www. forbes. com when ad blocker is on. As reported, a large portion of the web, 6.7\% of Alexa top 5000 [47] and $16.3 \%$ of the top 1000 popular live streaming sites [51] use this anti-adblocking system.

Besides, content-blocking can also lead to site breakage and other undesired app functionality [40]. This breakage can range from a dysfunctionality in part of the website (e.g., not displaying login popup) to the breakdown of the entire website layout. For instance, figure 4 shows a snapshot of www. store.vmware.com when Mozilla's ad and tracking protection is turned on. Furthermore, a large number of websites employ ad blocking circumvention to evade from ad blocking. For instance, www. thoughtcatalog. com and www.cnet.com obfuscate advertising URLs when they detect that the ad blocker is on. As a result, the resources are translated to the local servers and eventually displayed on the page. In all of the above cases, performance analysis of ads through ad blocking is infeasible which limits its scope.

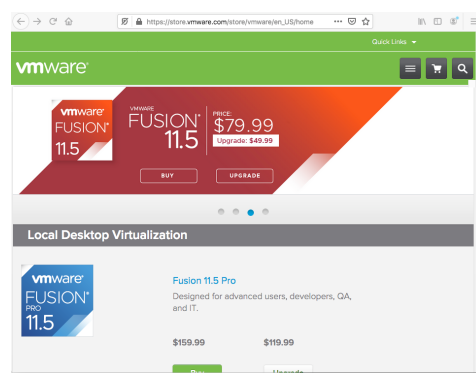

(a) Before content blocking

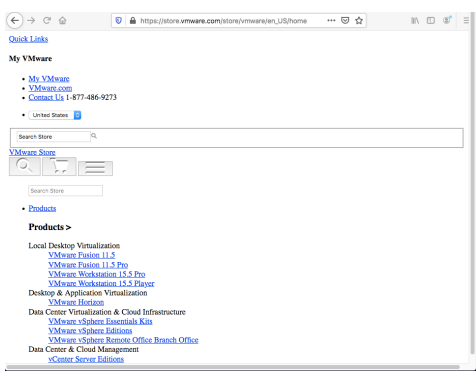

(b) After content blocking

Fig. 4. Snapshots of www. store.vmware.com. The layout of the page is broken due to content blocking.

Fine-grained analysis. Ad blockers block content as early as the initiation of network requests, which results in two drawbacks. First, it prevents fine-grained performance analysis at the browser level because activities such as content parsing and rendering related to the blocked ad become invisible for analysis. Hence, the current body of work focuses on the network data traffic overhead, 
neglecting the in-browser computation overhead of ads. Second, because the content is blocked at the network request, resources that are further requested by the blocked document during page loading become invisible for inspection. For example, when an ad exchange ${ }^{1}$ (e.g., Google AdSense) script is blocked, the source(s) of the blocked ads is hidden.

Our approach addresses the above limitations and enables an in-depth performance analysis of ads without adding significant overhead. As a result, our measured cost is more reliable and reveals some anomalies with previous studies that we discuss in section 7 . Plus, our framework measures performance cost of ads on every website. This is highly substantial in term of functionality because we examine only $15 \%$ of our test corpus ( $\approx 50$ websites) and discover 10 websites to have one of the discussed issues with ad-blocker. Ultimately, we do not block any content, this gives us the ability to correlate the performance cost of ads to the sources (domain analysis) as well as break down the computation cost at a finer level of granularity (browser activities) which have not been studied before. We present our first-of-a-kind findings from this fine-grained characterization in Section 6.

\subsection{Browser architecture}

A key to our performance characterization is measuring the amount of work the browser spends on loading the primary content of the page and the additional work in loading ads. Differentiating the two workloads (primary and ad content) requires an understanding of the way browsers load webpages. Here, we outline the browser's high-level design and workflow.

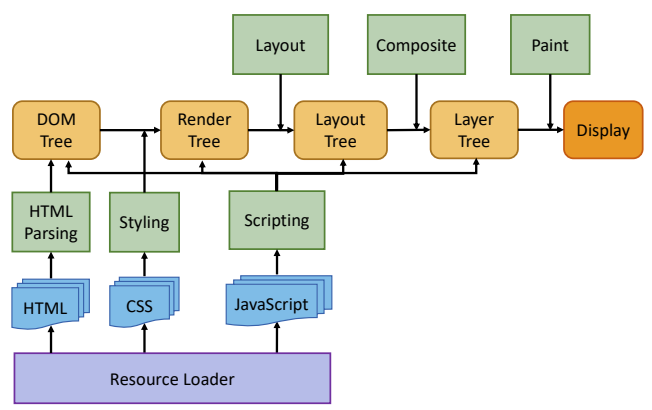

Fig. 5. High-level architecture of the web browser. The components include the resource loader (purple), six major computation stages (green), and the intermediate trees (yellow) in the page loading pipeline.

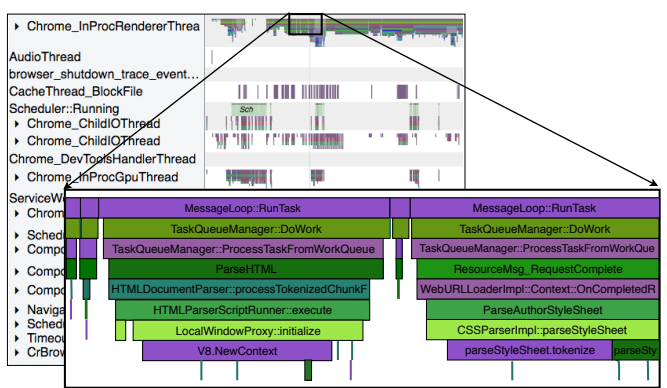

Fig. 6. Snapshot of the browser activities in loading www. apple.com that are executed on tens of threads. The zoom area shows the call stack (y-axis) of one thread over time (x-axis).

Modern browsers have different features and user interfaces but they are based on the same architecture to load webpages. Figure 5 shows the browser's high-level page loading workflow. The process begins when the user submits a URL request to the browser interface. The resource loader initiates HTTP requests and fetches resources (network activities) from the server. Once the resource is downloaded (incrementally or fully), the six major computation stages (shaded green) evaluate the resources and render the website. The computation stages are HTML parsing (builds the Document Object Model or DOM tree), Styling (evaluates stylesheets and adds attributes to the DOM tree), Scripting (responds to user interactions and dynamic behavior of the page), Layout (evaluates size and position of DOM elements), Composite (combines graphical layers), and Paint (maps layers to pixels). Each of these stages is composed of multiple sub-tasks. We will refer to

\footnotetext{
${ }^{1}$ A platform for buying and selling of advertising inventory from multiple ad networks through real-time bidding.
} 
these sub-tasks as activities in the rest of this paper. For example, the HTML parsing stage consists of byte stream decoding and preprocessing, tokenizing, and DOM tree construction activities.

The browser invokes the computation activities frequently during page loading. Figure 6 illustrates a snapshot of activities when loading www. apple.com. As we can observe, there are numerous and the order in which these activities execute is based on the dependency imposed by the page content. For instance, if JavaScript modifies an attribute of a DOM element, this forces the browser to recalculate the style, update layouts, composite layers, and repaint the screen. This is commonly known as reflow and can have a significant impact on performance. However, if the JavaScript only modifies the color of a DOM node, the reflow pipeline bypasses layout and if the change does not modify the graphical layers, the reflow also bypasses compositing. Moreover, browsers exploit parallelization between independent activities to accelerate page load time as seen in the figure. Due to the dependency between activities, dynamic parallelization, and stochastic behavior of the browser in resource downloading and dispatching tasks, the time of each activity is indeterminate. Therefore, tracking the dependency chain between browser activities and associating them to the corresponding workload type, i.e., ads or non-ads, are challenging.

\section{METHODOLOGY AND adPerf}

To distinguish the performance cost of web ads from the primary content (non-ads), we apply a systematic approach. First, we extract all browser activities that are associated with the page loading process. Second, we identify which resource (i.e., a web document) explicitly or implicitly initiates each browser activity. Third, we classify activities into ads and primary content based on the resource type initiating the activity. Finally, we measure the total execution time spent on each class of activity as a performance index distinguishing the workload in each class.

To realize the above methodology, we design and implement a tool, adPerf, for the Chrome browser. Note that adPerf can be extended to support other browsers since the same technique applies to all browser architectures. Figure 7 shows the design of adPerf. Below, we describe the four modules of adPerf - crawler, parser, resource mapper, and graph builder in detail.

\subsection{Crawler}

The first module in adPerf (top of the figure) is a crawler (Node.js script) that sets up the headless Chrome and crawls websites. The crawler uses the Chrome remote protocol APIs [8] under the hood to interact with the browser and streams Chrome traces [21] to a file. Chrome traces are primarily used for profiling and debugging the Chrome browser and are low-overhead. Tracing macros cost a few thousand clocks at most [21], and the logging to file happens after the page is loaded. Chrome traces are capable of recording intermediate browser activities, including page loading activities in the Blink rendering engine and V8 JavaScript engine with microsecond precision. Each trace contains information about the associated activity, such as thread id, activity name, function arguments, etc. Below is an example trace for a Scripting activity:

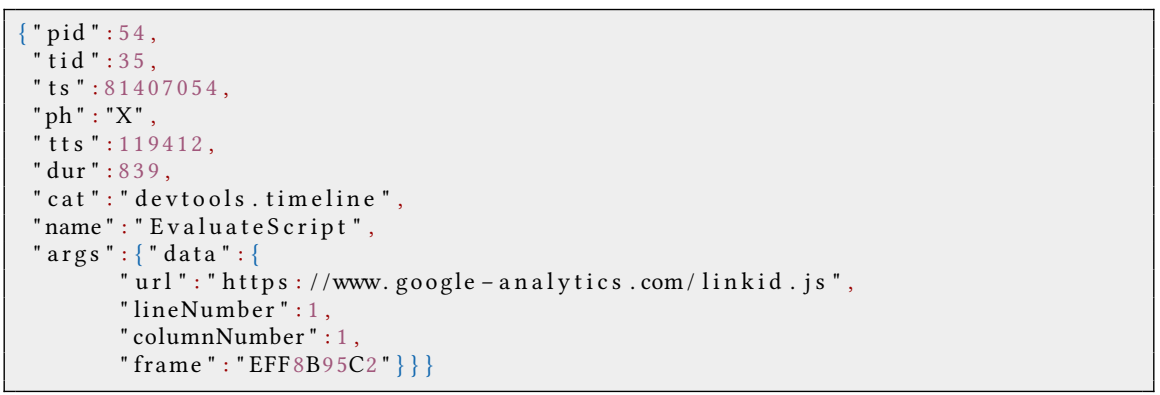


Additionally, the crawler intercepts network requests, i.e., onBeforeRequest event, and extracts the header and body of every HTTP request. This data is necessary for resource matching.

\subsection{Parser}

When the website is loaded, the raw Chrome traces are fed to the parser as shown in the figure. The adPerf parser does two tasks - pruning and data extraction.

Pruning. The parser goes through the traces and extracts all page loading activities and prunes the browser-dependent ones (such as browser garbage collection and inter-process communication activities). We use the same subset of traces that robust tools such as Chrome devtools timeline [9], Google Lighthouse [17], and COZ+ [48] collect for performance analysis and page loading workload characterization. The resulting activities are associated with one of the six browser stages shown in Figure 5. For instance, the parser considers every trace connected to script evaluation, V8 script compiling, V8 execution, callback functions triggered by browser events (or timeouts) among others as part of the Scripting stage.

Data extraction. For each activity, the parser extracts the following data: start time, end time, relative stage, thread and process ids, and function arguments if they contain resource information. This data is necessary to construct the call stack and attribute activities to resources.
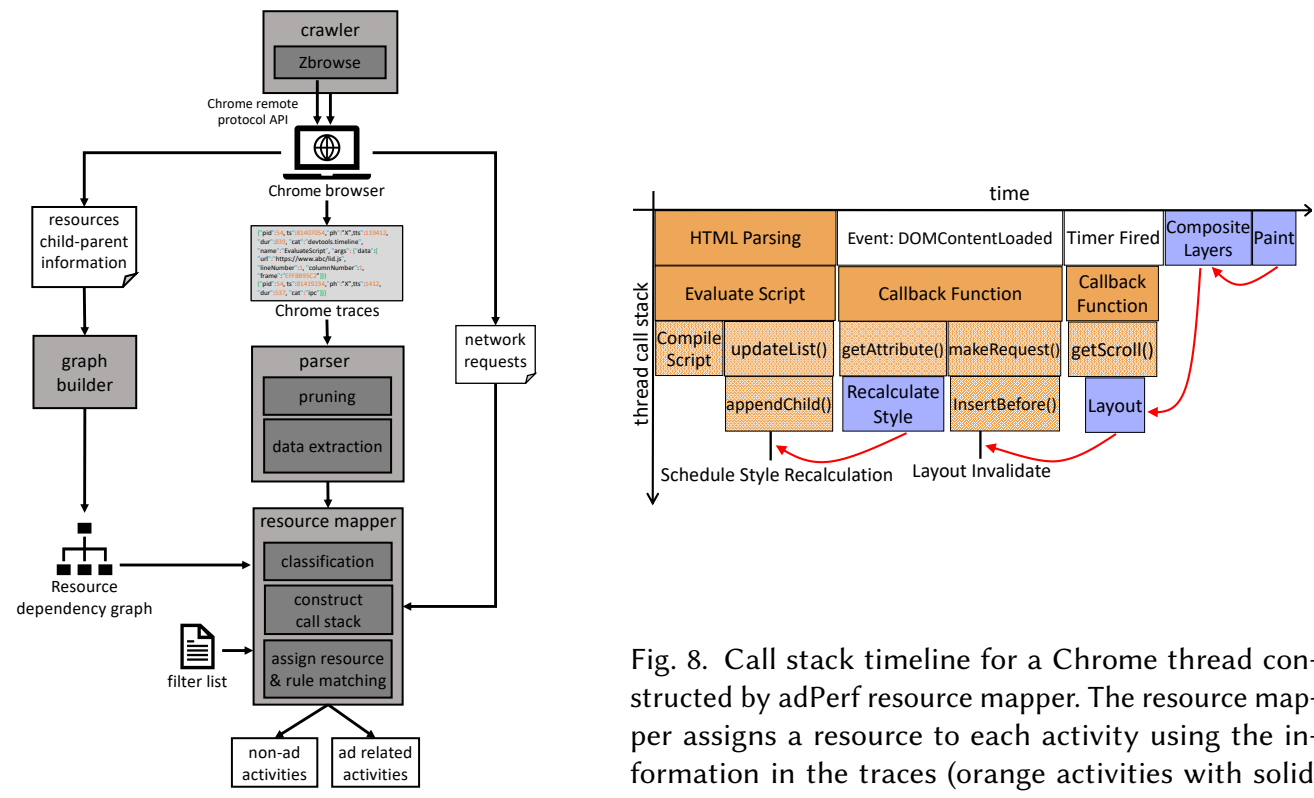

Fig. 8. Call stack timeline for a Chrome thread constructed by adPerf resource mapper. The resource mapper assigns a resource to each activity using the information in the traces (orange activities with solid texture) and call stack (orange activities with dotted

Fig. 7. Design of adPerf. The four core modules are texture) for parsing and evaluation activities and tracks crawler, parser, resource mapper, and graph builder initiator for tree manipulation and rendering activities that are shown with dark boxes. (purple activities)

\subsection{Resource mapper}

Once the traces are parsed and categorized, this data and network information extracted by the crawler are input to the resource mapper. The task of the resource mapper is to assign each activity to an associated resource. Unfortunately, we observed that a significant number of traces (about $30 \%$ ) do not contain any resource information. In such cases, the resource mapper has to derive this relation. 
To address the above challenge, the resource mapper first builds a call stack of activities for every thread by tracking the start time and end times of activities executed by each thread. Figure 8 shows the call stack timeline for a sample activity for a browser thread where activities are shown with boxes. After constructing call stacks, the resource mapper classifies activities into two groups - parsing and evaluation and tree manipulation and rendering. The former contains activities that explicitly relate to a resource such as HTML parsing, image decoding, stylesheet parsing, and JavaScript evaluation that directly operate on a document. Activities belonging to this group are colored orange in the figure. The latter contains activities that implicitly relate to a resource. These include activities in styling (except stylesheet parsing which belong to the former group), layout, composite, and paint stages that deal with the browser's intermediate data structures (trees) and display. Purple activities in the example belong to this group. Finally, the resource mapper finds the corresponding resource for each activity group as follows.

Parsing and evaluation. For the majority of the activities in this group, the resource mapper extracts the resource file information from function parameters extracted by the parser. Orange activities with solid texture such as HTML Parsing and Callback Function in Figure 8 are examples of activities where we can determine the document on which they parse or evaluate from frame id and resource information in their traces. However, a small number of activities in this group do not contain any resource information. For activities with unresolved resource files (activities shown with an orange color and dotted texture in the figure), the resource mapper uses the constructed call stack and follows their ancestors and associates them with the caller's resource file. For example, appendChild JavaScript function is called by updateList and this function along with Compile Script activity are invoked by Evaluate Script activity that is previously assigned to a JavaScript document.

Tree manipulation and rendering. For this group, we have to distinguish between the different resources that implicitly trigger the activities that belong to this group. For styling activities, we observe that Chrome recalculates styles after the Schedule Style Recalculation event is fired. As seen from Figure 8, this event is fired in the middle of parsing and evaluation of a resource (typically a JavaScript document) that attempts to modify the DOM node style. We track the call stack for this event to the initiated parsing and evaluation activity and associate this styling activity to the triggered document. Similarly, for layout, Chrome updates the layout tree when the Layout Invalidate event is fired. In our example, this is fired when the command this._util.elem.innerHTML $=e$ is executed in the InsertBefore() function. We use a similar procedure as styling to associate layout activities to the initiating resource from the call stack of the Layout Invalidate event.

Note that the browser does not always update the style and layout of nodes immediately after the events are triggered. Depending on the priority of other activities in the task scheduler queue, the browser might dispatch these activities later. As a result, when a resource triggers one of these two events (Schedule Style Recalculation or Layout Invalidate), a second resource may fire these events again before the browser updates the tree. In this case, we consider the first resource as the initiator since the tree will be traversed and updated even in the absence of the second resource. Chrome tends to composite and/or paint immediately after styling or layout which leads to repaint. Therefore, the associated resource for the composite and paint activities simply derives by following the chain to the last executed styling or layout activity as shown by the red arrows in the figure.

Once page loading activities are mapped to the corresponding resources, adPerf uses network data from the crawler to link the resources to the associated network requests (i.e., URLs). Then it uses a filter list to distinguish between ad resources and non-ad resources. We use EasyList [11], the primary and most popular filter rules list for advertisements for our experiments. However, users can also provide their own custom filter rules. adPerf employs adblockparser (an optimized python package [5]) to match the URLs against filter rules. One might think that since our methodology uses an identical rule matching procedure to ad blockers, it might incur a similar overhead. However, this 
is not the case since rule matching in adPerf is passive and does not steal computation cycles from the page loading process. Finally, adPerf reports the execution time of the page loading activities categorized by ads and non-ads.

\subsection{Graph builder}

There exist dependencies between resources on the website. For instance, let's say a website downloads a JavaScript file from a third-party domain. In this file, it can further request an image or an HTML document from another domain, and this chain can go deeper. To evaluate the performance cost of different sources such as ad domains and to further evaluate their trustworthiness requires first tracing this resource dependency chain and building a resource dependency graph.

We extract the dependency between resources of the websites using Zbrowse [25]. Zbrowse uses Chrome devTools protocol and allows us to instrument, inspect, and debug the Chrome browser. It also generates the child-parent relation for every network request. We embed Zbrowse in the adPerf crawler module as shown in Figure 7. This way, we can extract the resources child-parent data at the same time when we crawl the websites. The graph builder uses Zbrowse's output and constructs the dependency graph for resources. In cases where third-party JavaScript gets loaded into a first-party context and makes an AJAX request, the HTTP referrer appears to be the first-party. We follow [39] and allow the graph builder to conserve this relation and include the URL of the third-party from which the JavaScript was loaded. Since one resource can, in turn, request multiple resources, the constructed graph has the shape of a tree rather than simple chains of dependencies.

Figure 11 shows this graph for an example website, www. cnn. com. Here, we combine the resources from the same domain (at each level) into one node for easier visualization. The root node is the publisher and the remaining nodes are referred to as third-party domains. For differentiation, we color ad nodes (domains that deliver at least one ad resource) red and non-ad nodes (domains without any ad resources) blue in this graph. As we can see from the figure, a considerable number of third-party domains are ad nodes. This is a concerning finding since typically publishers are not aware of the contents delivered by third-party websites. Generally, publishers trust the first-party domains (in the first-level of the tree) but those websites might deliver their contents from another website or chain of websites that are not verified by the publishers. We investigate the prevalence of such third-party ad domains, their performance cost, and trustworthiness in section 6 .

\section{VALIDATION OF adPerf}

adPerf is a first-of-a-kind performance analyzing tool that measures the fine-grained performance overhead of web ads at the granularity of the browser's major stages. In the absence of tools with similar functionality to serve as a baseline, it is challenging to test and validate adPerf. Chrome DevTools [9], a set of web developer tools built directly into Google Chrome, provides sufficient and useful profiling data, including a breakdown of the browser workload into stages. However, the caveat is that the reported breakdown is for the entire page content, and it does not differentiate between ads and main content. Therefore, we devise the following experiment to exploit Chrome DevTools to calculate the performance of ads on a webpage and validate adPerf.

In our validation experiment, we first measure the total workload of a test page with Chrome DevTools. Then we instrument the test page by cloning every ad element on the page and re-measure the total workload. If the cloning is perfect, the added workload will present the performance overhead of web ads. This experiment validates two main objectives:

(1) How precisely does adPerf measure the computation workload (irrespective of ads and main content) and classify them by the browser stages? This is achieved by comparing adPerf's reported total workload and its breakdown with Chrome DevTools data. 


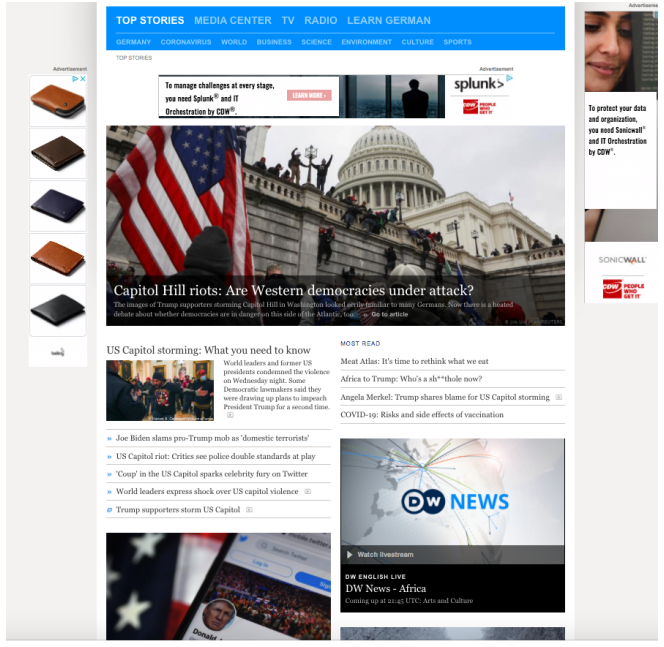

Fig. 9. Snapshot of www.dw.com before cloning ads.

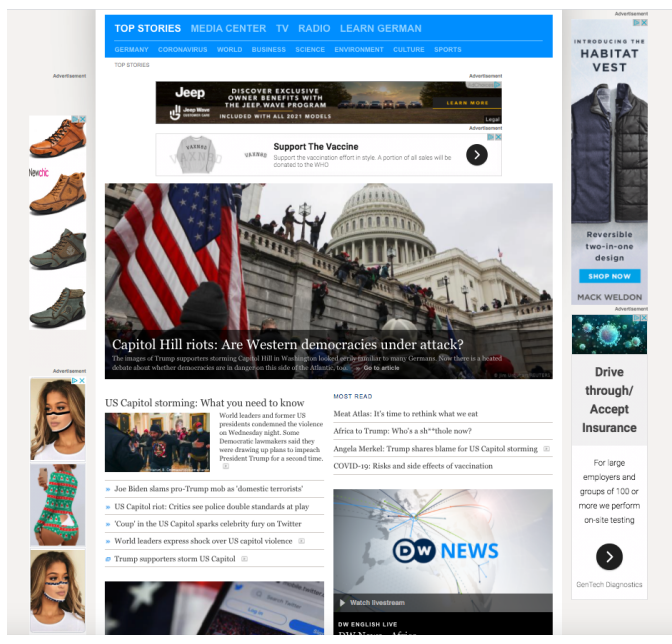

Fig. 10. Snapshot of www.dw.com after cloning ads.

(2) More important, how well does adPerf distinguish the main content workload from the advertising workload? This is validated by comparing the performance of the added workload (which represents only ads) measured by Chrome Devtools with adPerf's reported ads performance cost.

Instrumenting real-world websites is comparatively more arduous than synthetic pages due to the complexity and obfuscation of page sources. However, we adhere to the former for the sake of proximity to in-the-wild ads and fairness in our validations. Moreover, we duplicate every ad element (including leaderboards, infeed ads, sticky and animated banners, etc.) and do not limit ourselves to a specific type of ad for completeness. Without bias, we randomly pick five websites from our test corpus (see Section 5) and instrument them. Figures 9 and 10 show an example of this instrumentation on the appearance of a website where we observe that ads are duplicated. Note that ads are typically delivered from a bidding system (i.e., ad exchange) thus, a duplicated ad is not necessarily identical to the original ad. However, to minimize the impact of this stochastic behavior, we load websites multiple times and include cases where two ads (original and cloned) have at least the same structure and size.

Table 1 summarizes the results from the validation tests on the accuracy of adPerf in measuring the page loading workload and fine-grained breakdown by browser stages on the original webpage. We observe that adPerf measures total page-dependent browser computation within $0.4 \%$ to $6.8 \%$ of Chrome DevTools for the five randomly sampled test webpages. Besides, adPerf's breakdown is well in line with Chrome DevTools, and all the stage measurements are below a $12 \%$ margin of error, with a median error of 5\%. This verifies adPerf's parser, pruning, call-stack construction, and activity classification are functioning accurately.

In Table 2, we present our results from the performance dissection of ads by both adPerf and Chrome DevTools for the same websites. For each website, adPerf indicates ads performance cost reported by adPerf on the original page, and Chrome signifies the ads cost estimated by Chrome DevTools (which is measured by calculating the difference in timings between the original page and the page with duplicated ads). The results show that adPerf's total ad costs are within $11 \%$ of Chrome DevTools estimation which confirms that adPerf's graph builder, call-stack analyzer, activity tracker, and resource matcher modules are designed correctly, and adPerf successfully 
Table 1. Comparison of adPerf with Chrome DevTools in measuring the total computation time and breakdown by browser stages on a MacBook Air laptop.

\begin{tabular}{|c|c|c|c|c|c|c|c|c|c|c|c|}
\hline \multirow[t]{2}{*}{ Website } & & \multicolumn{2}{|c|}{ Total } & \multicolumn{2}{|c|}{ Parsing } & \multicolumn{2}{|c|}{ Scripting } & \multicolumn{2}{|c|}{ Rendering } & \multicolumn{2}{|c|}{ Painting } \\
\hline & & $\begin{array}{l}\text { Time } \\
(\mathrm{sec})\end{array}$ & $\begin{array}{c}\text { Error } \\
(\%)\end{array}$ & $\begin{array}{l}\text { Time } \\
\text { (sec) }\end{array}$ & $\begin{array}{c}\text { Error } \\
(\%)\end{array}$ & $\begin{array}{l}\text { Time } \\
\text { (sec) }\end{array}$ & $\begin{array}{c}\text { Error } \\
(\%)\end{array}$ & $\begin{array}{l}\text { Time } \\
\text { (sec) }\end{array}$ & $\begin{array}{c}\text { Error } \\
(\%)\end{array}$ & $\begin{array}{l}\text { Time } \\
\text { (sec) }\end{array}$ & $\begin{array}{c}\text { Error } \\
(\%)\end{array}$ \\
\hline \multirow{2}{*}{$\begin{array}{l}\text { newindian- } \\
\text { express.com }\end{array}$} & adPerf & 18.2 & & 1.06 & & 14.9 & & 1.91 & & 0.28 & \\
\hline & Chrome & 18.6 & 1.6 & 1.17 & 8.9 & 15.3 & 2.2 & 1.79 & 6.9 & 0.26 & 6.5 \\
\hline \multirow{2}{*}{$\begin{array}{l}\text { buffalonews } \\
\text {.com }\end{array}$} & adPerf & 12.2 & & 0.65 & & 9.33 & & 1.91 & & 0.36 & \\
\hline & Chrome & 12.6 & 2.9 & 0.58 & 10.9 & 9.88 & 5.5 & 1.83 & 4.5 & 0.33 & 12 \\
\hline \multirow[t]{2}{*}{ huffpost.com } & adPerf & 2.13 & & 0.13 & & 1.61 & & 0.30 & & 0.09 & \\
\hline & Chrome & 2.28 & 6.8 & 0.13 & $<1$ & 1.76 & 8.7 & 0.31 & 2.3 & 0.08 & 6.0 \\
\hline \multirow[t]{2}{*}{ observer.com } & adPerf & 3.12 & & 0.16 & & 2.40 & & 0.47 & & 0.09 & \\
\hline & Chrome & 3.43 & 0.41 & 0.16 & 0.43 & 2.72 & 0.49 & 0.46 & 0.57 & 0.08 & 0.57 \\
\hline \multirow[t]{2}{*}{ dw.com } & adPerf & 3.20 & & 0.25 & & 2.34 & & 0.51 & & 0.10 & \\
\hline & Chrome & 3.38 & 5.1 & 0.24 & 6.3 & 2.54 & 8.0 & 0.50 & 1.2 & 0.09 & 10.6 \\
\hline
\end{tabular}

Table 2. Comparison of the ads performance cost reported by adPerf with Chrome DevTools estimation on a MacBook Air laptop. adPerf indicates the ads performance cost reported by adPerf on the original page. Chrome indicates the ads cost estimated using Chrome DevTools by computing the difference in timing between the original page and the page with cloned ads. adPerf $2 x$ indicates the increase in ad workload reported by adPerf after cloning ads.

\begin{tabular}{|c|c|c|c|c|c|c|c|c|c|c|c|}
\hline \multirow[t]{2}{*}{ Website } & & \multicolumn{2}{|c|}{ Total } & \multicolumn{2}{|c|}{ Parsing } & \multicolumn{2}{|c|}{ Scripting } & \multicolumn{2}{|c|}{ Rendering } & \multicolumn{2}{|c|}{ Painting } \\
\hline & & $\begin{array}{l}\text { Time } \\
(\mathrm{ms})\end{array}$ & $\begin{array}{l}\text { Err. } \\
(\%)\end{array}$ & $\begin{array}{l}\text { Time } \\
(\mathrm{ms})\end{array}$ & $\begin{array}{l}\text { Err. } \\
(\%)\end{array}$ & $\begin{array}{l}\text { Time } \\
(\mathrm{ms})\end{array}$ & $\begin{array}{l}\text { Err. } \\
(\%)\end{array}$ & $\begin{array}{l}\text { Time } \\
(\mathrm{ms})\end{array}$ & $\begin{array}{l}\text { Err. } \\
(\%)\end{array}$ & $\begin{array}{l}\text { Time } \\
(\mathrm{ms})\end{array}$ & $\begin{array}{l}\text { Err. } \\
(\%)\end{array}$ \\
\hline \multirow{3}{*}{$\begin{array}{l}\text { newindian } \\
\text { express.com }\end{array}$} & adPerf & 2750 & & 134 & & 2470 & & 131 & & 22 & \\
\hline & Chrome & 2560 & 7.1 & 120 & 10.4 & 2270 & 7.9 & 142 & 12 & 25 & 3.1 \\
\hline & $\operatorname{adPerf} 2 \mathrm{x}$ & 2840 & 3.1 & 146 & 8.2 & 2540 & 2.9 & 134 & 4.5 & 21 & 7.1 \\
\hline \multirow{3}{*}{ buffalonews.com } & adPerf & 635 & & 91 & & 411 & & 121 & & 12 & \\
\hline & Chrome & 683 & 7.0 & 84 & 7.7 & 478 & 14 & 111 & 8.3 & 10 & 23 \\
\hline & $\operatorname{adPerf} 2 \mathrm{x}$ & 520 & 15 & 81 & 11 & 349 & 15 & 94 & 22 & 16 & 19 \\
\hline \multirow{3}{*}{ huffpost.com } & adPerf & 694 & & 56 & & 432 & & 163 & & 43 & \\
\hline & Chrome & 671 & 3.3 & 54 & 3.6 & 413 & 4.4 & 157 & 3.7 & 47 & 8.5 \\
\hline & $\operatorname{adPerf} 2 \mathrm{x}$ & 730 & 4.9 & 59 & 5.0 & 458 & 5.7 & 168 & 3.0 & 45 & 4.4 \\
\hline \multirow{3}{*}{ observer.com } & adPerf & 400 & & 17 & & 258 & & 117 & & 8 & \\
\hline & Chrome & 451 & 11 & 19 & 10.5 & 316 & 18.4 & 107 & 8.5 & 9 & 0.41 \\
\hline & $\operatorname{adPerf} 2 \mathrm{x}$ & 352 & 12 & 16 & 5.9 & 225 & 12.8 & 104 & 11.1 & 7 & 12.5 \\
\hline \multirow{3}{*}{ dw.com } & adPerf & 1040 & & 85 & & 721 & & 196 & & 38 & \\
\hline & Chrome & 971 & 6.6 & 93 & 8.6 & 641 & 11.1 & 190 & 3.1 & 44 & 13.6 \\
\hline & $\operatorname{adPerf} 2 \mathrm{x}$ & 870 & 16 & 85 & $<1$ & 684 & 5.1 & 172 & 12.2 & 44 & 13.6 \\
\hline
\end{tabular}

isolates ads from the main content. Additionally, using adPerf, we measure the increase in the total page workload after cloning ads which are denoted by adPerf $2 x$ in Table 2 . We then compare the former against the cost of the original ads for each website. This comparison shows that duplicating ads does not precisely double the performance cost of ads but is within an acceptable range of $3.1 \%$ to $16 \%$ of the original ads cost. The inaccuracy primarily stems from the fact that the cloned ads do not exactly resemble the original ads as seen from Figures 9 and 10 due to the bidding system. We attribute the marginal errors in the validation against Chrome DevTools to the same artifact and anticipate adPerf's reported ad cost to be even closer to reality. 


\section{EXPERIMENTAL SETUP}

System. Our system is a MacBook Pro with 2 cores and 8 GB RAM connected to a high-speed WiFi (400 Mbps). The mobile experiments are conducted on a Nexus 6P (quad-core ARM Cortex-A53 + quad-core ARM Cortex-A57 processor) connected to the cellular network. To obtain accurate results on communication overhead, we do not set up any proxy or local server.

Test corpus. Our test corpus consists of two sets of web pages - (a) top 350 websites from Alexa top 500 news list [6] and (b) top 200 websites from Alexa top 500 list [7]. We will refer to these two web page datasets as news and general respectively. The two lists have only 17 websites in common. For each dataset, we crawl the corresponding corpus twice. The first time, we crawl the home page or landing page of the website. The second time, we randomly click a link on the home page and crawl the page that it leads to. We exploit Chrome Popeteer [20] to automate link clicking. We refer to the former as the landing page crawl and the latter as the post-click page crawl.

Experimental repeat. In each crawl over the corpus (total 4 crawls), we load websites multiple times and take the average to account for fluctuations in page loading.

Evaluation domain. Since the main goal is to characterize the performance cost of ads, we primarily provide evaluation results for the websites that contain ads. This is nearly $80 \%$ of news websites and $40 \%$ of top general websites.

\section{RESULTS AND DISCUSSION}

In this section, we analyze the performance cost of ads from two viewpoints - at the ad domains (close to the origin) and deeper in the browser (close to the metal). First, using adPerf, we analyze the performance cost of ads on the websites broken down by costs incurred by the computation (i.e., rendering engine) and network (i.e., resource loader). Then, we investigate a level deeper to understand which computation stages and network resources mainly contribute to the computation and network ad costs respectively. Finally, we zoom out and analyze the ad domains themselves to quantify their contribution to the performance cost of web ads.

\subsection{Computation cost of ads}

For every website, we calculate the fraction of time spent in ad-related activities to the total activities $(\mathrm{ad}+$ non-ad). Figure 12 shows the CDF distribution of this fraction for the 4 different crawls.

Finding 1. According to the figure, web ads can have a significant impact on the performance of the website. For example, half of the news websites spend more than $15 \%$ of their computing time on ads. Moreover, $20 \%$ of the news websites spend more than $30 \%$ of the time on advertising which can be concerning from the user's perspective. It also motivates website builders and ad providers to optimize their advert contents. Compared to the news websites, ads have a lower cost on the general corpus. The median in this corpus is $5 \%$.

Finding 2. The figure presents another interesting detail when we compare the landing and post-click page graphs. Ads have a higher performance cost when loading the landing page versus the post-click page of news websites by about $25 \%$ on average. However, this is not the case for general websites. Post-click pages of popular general websites have almost similar cost-performant ads as the landing page. Further, we aggregate the total time spent on ad-activities across all browser stages and compare that to the time spent on the main content. The average percentage of time spent on ads versus main content for the news landing page, news post-click, general landing page, and general post-click datasets is $17,15,11$, and $10 \%$ respectively. The averages are higher than the median percentages reported earlier because a small number of websites spend $40-50 \%$ of the computation time on ad-activities. 


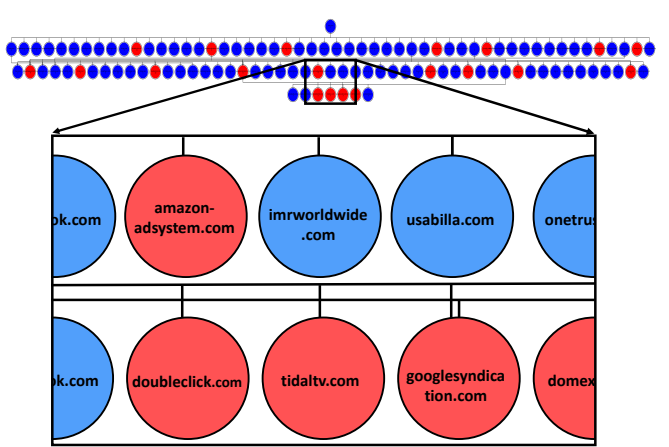

Fig. 11. Resource-dependency graph for www.cnn.com. Ad nodes are colored red and non-ad nodes are colored blue.

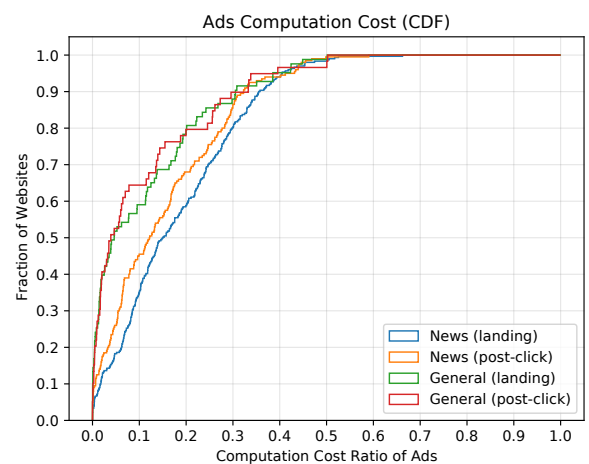

Fig. 12. Computation cost of ads in two datasets namely top general and top news websites. Each domain in the dataset is crawled twice (landing page and post-click page).

Breakdown of ad computation cost. Since we observe that ads can have a significant impact on website loading, it is worthwhile to explore the cause of this overhead. This can guide website builders and ad providers to focus their optimization efforts on those activities that are the primary sources of performance loss. Accordingly, we classify the computation cost of ads by the granularity of the browser stages (outlined in Section 2). Figure 13 shows the contribution of the six major stages for the news corpus. For each stage, $s$, we measure the following three metrics. Note that $c t^{s}$ is the computation time of stage $s$ while $c t^{*}$ is the total time spent in computation across all the stages. Similarly, $c t_{a d}$ is the computation time spent on ad-activities while $c t_{*}$ is the total time spent on all activities. Therefore, $c t_{*}^{*}$ is the total time of all computation activities in the browser.

(1) The fraction of time spent on ad-activities in stage $s$ to the total time spent on all activities in stage $s\left[c t_{a d}^{s} / c t_{*}^{s}\right]$. This is shown by the green bars. This intra-stage metric indicates how the workload of the stage, $s$, is split between ads and the main content.

(2) The fraction of time spent on ad-activities in stage $s$ to the total time spent on ad-activities across all stages $\left[c t_{a d}^{s} / c t_{a d}^{*}\right]$. This is shown by the blue bars. This inter-stage highlights how a particular stage, $s$, is impacted by ads compared to the other browser stages.

(3) The fraction of time spent on all activities in stage $s$ to the total page load computation time $\left[c t_{*}^{s} / c t_{*}^{*}\right]$. This is another inter-stage metric shown by the red bars. However, unlike the above metric, it shows the influence of a particular stage, $s$, on the entire page load.

It is important to correlate both the inter-stage metrics to have a complete analysis. For example, if a stage has a significant contribution to ads (i.e., second metric) but has very little impact on page loading (i.e., third metric), then it is unlikely to be a performance optimization target.

Finding 3. Figure 13 shows that scripting has the highest impact, more than $88 \%$, on the computation cost of ads. It also has a significant impact (73\%) on the computation workload of the entire page. The difference between these two metrics indicates that ads are more scripting heavy than the main content. This is because ad-content has $21 \%$ more dynamic characteristics than the original page content in our news corpus which increases the time spent in scripting. However, scripting only spends $25 \%$ of its time on ad-related content (i.e., first metric). Therefore, ads are not the primary bottleneck of the scripting stage but optimizing this stage will considerably improve the performance of ads as scripting is the major workload of today's web ads on news sites. 

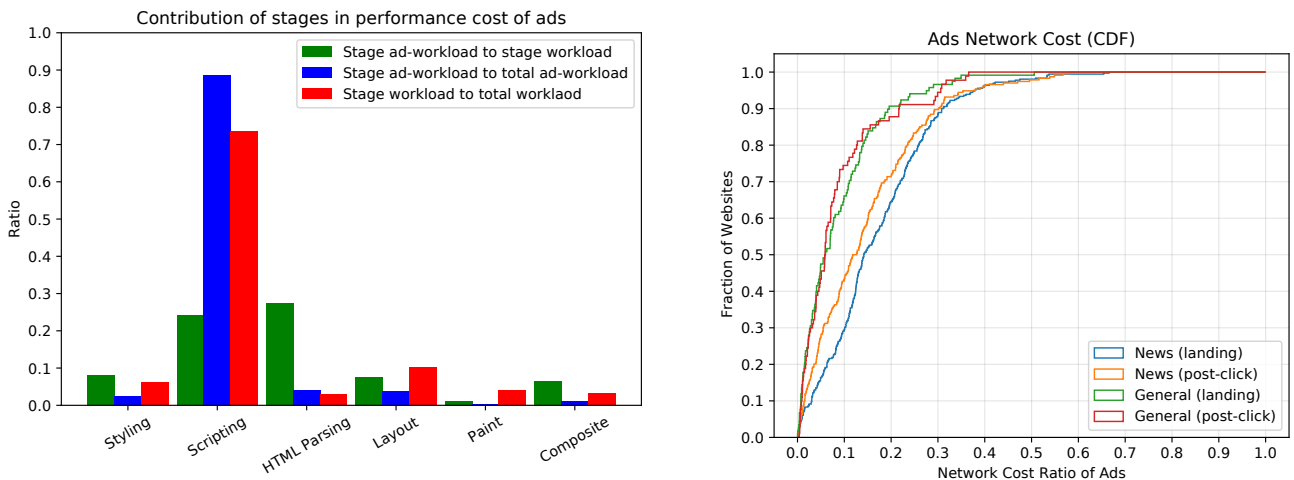

Fig. 13. Contribution of the different browser stages to the performance cost of ads for the news landing corpus. The three bars for each stage correspond to the three ratio metrics $\left(c t_{a d}^{s} / c t_{*}^{s}, c t_{a d}^{s} / c t_{a d}^{*}\right.$, and $\left.c t_{*}^{s} / c t_{*}^{*}\right)$.

Fig. 14. Network performance cost of ads in two corpuses: general and news websites. Each corpus contains landing and post-click pages.

Finding 4. Another observation from Figure 13 is that HTML parsing has a minor influence on page loading, i.e., less than $5 \%$ in comparison with scripting but ads have more impact on this stage (comparing green bars). In other words, optimizing ads HTML code is expected to improve HTML parsing workload more than optimizing ads JavaScripts for the scripting stage, even though HTML optimizations can only marginally improve page load time. This underscores the importance of correlating the intra- and inter-stage metrics to guide optimization efforts. We observe similar behavior for the general corpus as well.

\subsection{Network cost of ads}

Besides computation activities, loading ads imposes overhead on the network activities. To measure the performance cost of ads over the network, for each website, we calculate the ratio of time spent on fetching ad-related resources to the total time spent on fetching all the requested resources. Figure 14 shows the CDF of this network cost ratio for the 4 crawls.

Finding 5. The four distributions follow the same order as in Figure 12 (computation cost of ads), i.e., news websites incur higher network performance cost than general websites. This is not surprising since more and/or larger ad resources also require more work in parsing, evaluating, and rendering. According to the figure, the median of the network-cost ratio is $15 \%$ for news websites' landing page and $3 \%$ less on the post-click page. For the general websites, the median is $6 \%$ for the landing page and post-click page respectively.

Breakdown of ad network cost. To dissect the network costs of ads, we breakdown the network time consumption by content type (such as HTML, image, and media). For each content type, Table 3 summarizes statistics about the frequency of resources and network time spent on fetching those resources for the news corpus for both landing and post-click pages. Given the number of resources, $n r$, and network time spent on the resources, $n t$, we define three metrics for each (similar to computation stages) as follows.

- Metrics for the number of resources $(n r)$.

(1) The fraction of the number of resources of content type, $c$ to the total number of resources of $c\left[n r_{a d}^{c} / n r_{*}^{c}\right]$ (intra resource-type metric). 
Table 3. Summary of the three metrics each for the number of resources and network time spent on resources across two types of pages (landing page denoted by $L$ and post-click page denoted by PC) for the news corpus.

\begin{tabular}{|c|c|c|c|c|c|c|c|c|c|c|c|c|}
\hline \multirow{3}{*}{ cont. type } & \multicolumn{6}{|c|}{ Stats for the number of resources } & \multicolumn{6}{|c|}{ Stats for the request time of resources } \\
\hline & \multicolumn{2}{|c|}{$n r_{a d}^{c} / n r_{*}^{c}$} & \multicolumn{2}{|c|}{$n r_{a d}^{c} / n r_{a d}^{*}$} & \multicolumn{2}{|c|}{$n r_{*}^{c} / n r_{*}^{*}$} & \multicolumn{2}{|c|}{$n t_{a d}^{c} / n t_{*}^{c}$} & \multicolumn{2}{|c|}{$n t_{a d}^{c} / n t_{a d}^{*}$} & \multicolumn{2}{|c|}{$n t_{*}^{c} / n t_{*}^{*}$} \\
\hline & $\mathrm{L}$ & $\mathrm{PC}$ & $\mathrm{L}$ & PC & $\mathrm{L}$ & $\mathrm{PC}$ & $\mathrm{L}$ & $\mathrm{PC}$ & $\mathrm{L}$ & $\mathrm{PC}$ & $\mathrm{L}$ & $\mathrm{PC}$ \\
\hline Script & 0.23 & 0.22 & 0.41 & 0.45 & 0.40 & 0.43 & 0.25 & 0.24 & 0.49 & 0.57 & 0.33 & 0.37 \\
\hline HTML & 0.36 & 0.34 & 0.09 & 0.09 & 0.05 & 0.06 & 0.17 & 0.14 & 0.04 & 0.05 & 0.04 & 0.05 \\
\hline Image & 0.23 & 0.22 & 0.37 & 0.35 & 0.37 & 0.33 & 0.13 & 0.12 & 0.39 & 0.32 & 0.50 & 0.42 \\
\hline Font & 0.13 & 0.06 & 0.01 & 0.01 & 0.02 & 0.02 & 0.06 & 0.03 & 0.01 & $<0.01$ & 0.02 & 0.02 \\
\hline CSS & 0.06 & 0.03 & 0.01 & 0.01 & 0.05 & 0.06 & 0.05 & 0.02 & 0.01 & 0.01 & 0.03 & 0.04 \\
\hline XML & 0.54 & 0.46 & 0.01 & $<0.01$ & $<0.01$ & $<0.01$ & 0.68 & 0.43 & 0.01 & $<0.01$ & $<0.01$ & $<0.01$ \\
\hline XHR & 0.18 & 0.12 & 0.04 & 0.03 & 0.06 & 0.05 & 0.12 & 0.07 & 0.05 & 0.04 & $\mathbf{0 . 0 7}$ & 0.08 \\
\hline Media & 0.04 & 0.04 & $<0.01$ & $<0.01$ & $<0.01$ & $<0.01$ & 0.03 & 0.03 & $<0.01$ & $<0.01$ & $<0.01$ & $<0.01$ \\
\hline Unknown & 0.24 & 0.30 & 0.05 & 0.05 & 0.05 & 0.04 & 0.06 & 0.13 & $<0.01$ & 0.01 & 0.01 & 0.01 \\
\hline
\end{tabular}

(2) The fraction of the number of ad-resources of content type, $c$ to the total number of ad-resources (of all content types) $\left[n r_{a d}^{c} / n r_{a d}^{*}\right]$.

(3) The fraction of the number of resources of content type, $c$ to the total number of resources $\left[n r_{*}^{c} / n r_{*}^{*}\right]$ to highlight the popularity of the content type.

- Metrics for the network time spent on resources $(n t)$.

(1) The fraction of the network time spent on ad-resources of content type, $c$ to the total network time spent on resources of $c\left[n t_{a d}^{c} / n t_{*}^{c}\right]$.

(2) The fraction of the network time spent on ad-resources of content type, $c$ to the total network time spent on ad-resources (of all content types) $\left[n t_{a d}^{c} / n t_{a d}^{*}\right]$.

(3) The fraction of the network time spent on resources of content type, $c$ to the total network time spent on all resources $\left[n t_{*}^{c} / n t_{*}^{*}\right]$ to accent the performance impact of content type, $c$.

For instance, the first metric for network time of CSS refers to the fraction of time spent on fetching ad-related CSS resources to the time spent on fetching all CSS resources $\left[n t_{a d}^{c s s} / n t_{*}^{c s s}\right]$.

Finding 6. Among all content types, Table 3 shows that XML has the largest percentage of ad resources for both landing (54\% which account for $68 \%$ of the network time in fetching XML resources from metric 1) and post-click pages (46\% which take up $43 \%$ of the network time). However, it contributes to an insignificant fraction of the network performance cost for both pages (metric 2). On the contrary, scripts and images commonly used by ad providers, make up nearly $80 \%$ of all ad resources (metric 2) and all resources (metric 3) for both landing and post-click pages. Among the two content types, scripts on average are $20 \%$ more popular than images for post-click pages compared to the landing page (comparing metrics 2 and 3). Script files used in advertising alone are responsible for almost half of the network performance cost of ads, followed by images at $40 \%$ for landing pages (metric 2). More scripts in post-click pages correspond to a higher contribution to the network time spent in ads (57\%) for these pages compared to images (33\%).

Finding 7. Ad-related HTML files constitute $34-36 \%$ of total HTML files but they only take $14-17 \%$ of download time. A deeper investigation shows that ad HTML documents are lighter than main-content HTML. The former has a significantly small number of tags (on average 7) including only one or two <script $>$ tags that encapsulate small and minified code compared to the main-content HTML files with 410 tags. Surprisingly, XHR (XMLHttpRequest) resources make up

Proc. ACM Meas. Anal. Comput. Syst., Vol. 5, No. 1, Article 3. Publication date: March 2021. 
a significant $7 \%$ of the network performance cost for the landing page and $9 \%$ for post-click pages (metric 3). The corresponding time spent on ad resources is $5 \%$ and $4 \%$ respectively (metric 2).

\subsection{Breakdown of ad performance by source}

The results so far breakdown the performance cost of web ads at the lower level of granularity. Now, we zoom out and quantify the cost of ads based on their origin, i.e., ad domains. The goal of this lens is to gain an understanding of the third-party ad domains and their impact on the performance cost. Accordingly, we build the resource-dependency graph (as described in section 3) for all news websites in our test corpus. Overall we identify more than 300 distinct ad domains.

Breakdown of computation performance cost by ad domains. For every ad domain, we first aggregate the time the rendering engine spends on evaluating the resources served by that domain. We also measure the total time spent on ads through the crawl (ads computation cost). The ratio between the above two is an indicator of how each third-party ad domain contributes to the computation cost of ads. Figure 15(a) shows the contribution of the top 10 ad domains (out of 300) in decreasing order (from left to right) of their performance impact. The number on top of each bar is the number of websites in our corpus that are served by that ad domain.

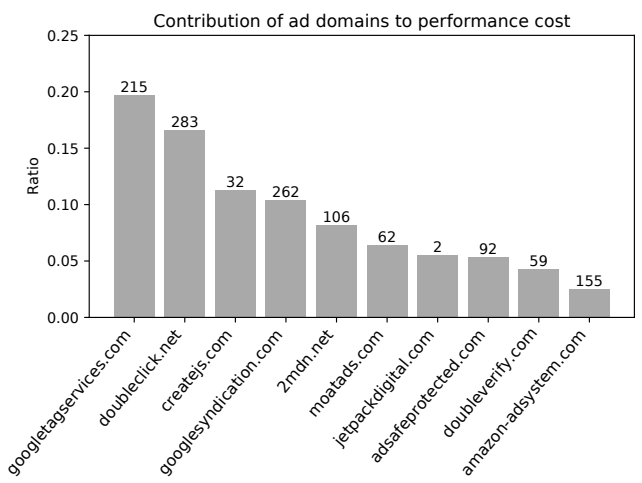

(a) Computation

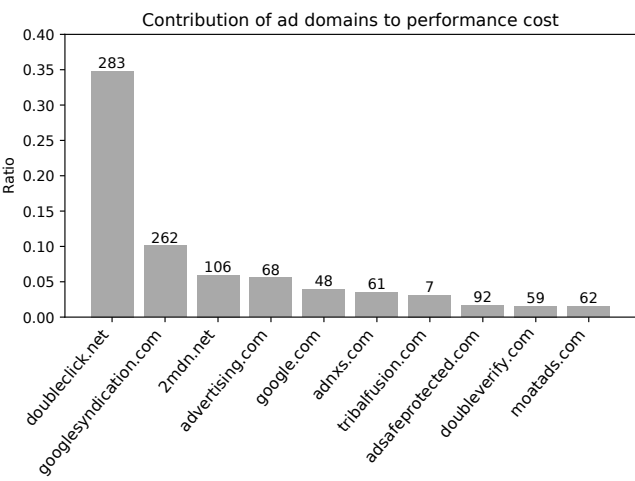

(b) Network

Fig. 15. Contribution of ad domains to the performance cost of web ads. The number on top of each bar is the number of websites serviced by that particular ad domain.

Finding 9. googletagservices. com and doubleclick. net have the highest contribution to the computation of ads on the web. The former is a Google tag management system for managing JavaScript and HTML tags used for tracking and analytics on websites, and the latter is a popular ad provider. Together, they deliver about $35 \%$ of the total ad resources. Moreover, all the ads are not delivered by well-known ad domains. In our corpus, $50 \%$ of ad domains appear only in the dependency graph of one website.

Besides, the number of websites serviced by an ad domain is not an indicator of its performance cost. For instance, googlesyndication. com has approximately the same contribution to the performance cost of ads as createjs. com but it services over $8 \times$ more websites than the latter. This is because createjs. com provides content for interactive ads (flash-like ads using HTML5 canvas) that trigger JavaScript callback functions constantly to sporadically change the content and re-flow. createJS ads (where usually incorporated by intermediate ad domain) on 32 websites of our corpus heavily use Scripting activities, $7.5 \%$ more than Scripting activities belong to googlesyndication. com on 262 websites. 
Breakdown of network cost by ad domains. We follow a similar procedure as above for estimating the contribution of individual ad domains to the network cost of a page load. For every ad domain, we first aggregate the time the browser spends on fetching resources by that domain, Then, we calculate the ratio of the total time spent on fetching ad resources in our crawl to the above time. Figure 15(b) shows the top 10 ad domains that have the highest contribution to the network cost of ads in the news corpus.

Finding 10. About $35 \%$ of the network cost of ads on news websites is traced to doubleclick. net followed by the popular ad syndication googleadsyndication. com with $10 \%$ contribution. Google is the major actor in the ad ecosystem. Domains maintained by Google alone constitute approximately $51 \%$ of the total ad network cost.

Finding 11. Comparing the computation cost of domains with their network cost shows that these two performance costs are correlated. As one might expect, fetching more and larger documents also takes longer to evaluate and display. Interestingly, we also observe domains that have a high computation cost but insignificant network cost and vice versa. For instance, googletagservices . com has the highest contribution (19.7\%) to the computation cost of ads among all 300 ad domains. However, it contributes to less than $1 \%$ of the network cost (ranked 16 and not shown in the top 10 domains in Figure 15(b)). Further breakdown of its performance cost with adPerf reveals two JavaScript documents (osd.js and osd_listener.js) of size less than $76 \mathrm{~KB}$ belonging to this domain referenced by over 200 websites in the news corpus. These two files are part of Google Ads that track the viewability of the ads to assess the value of an impression to the publisher and advertiser. To calculate what percentage of an ad appears in a viewable space on the screen and for how long that portion of the ad remains visible, these JavaScript snippets are frequently invoked by the webpage and take up valuable CPU cycles.

Breakdown of performance cost by trustworthiness. When a publisher displays an ad on their webpage, there is an explicit trust between the publisher and the provider. However, when the ad provider is part of a syndication, the ad is served through a chain of redirections going through different ad domains. Our measurement results on the Alexa news and general websites shows that the mean depth of this chain is $\mathbf{4}$, revealing ad syndication is prevalent. Most of the ad domains on the chain are not directly visible to the publisher (except the ones directly embedded by the publisher). As a result, the publisher cannot verify their intention (e.g., whether used for drive-by download or phishing). This results in the publisher placing an implicit trust in the ads since the trustworthiness of these ad domains is unknown. In this paper, we are interested in the correlation between the performance cost of an ad domain and its trustworthiness.

To this end, we leveraged two online services, WOT (Web of Trust) [24] and VirusTotal [23], to model the trustworthiness of an ad domain. WOT is a community-based reputation system that assigns a score to a domain name based on user complaints and other blacklists. The score ranges from 0 to 100, and WOT classifies domains based on their scores into 5 trust rating - excellent, good, unsatisfactory, poor, and very poor [31]. VirusTotal is a portal that proxies the request of a security check of a domain/URL to its affiliated blacklist services (71 blacklists). When a domain is submitted to VirusTotal, it reports the blacklists that flag it as red. We count the ratio of blacklists that do not raise an alarm on the domain (i.e., safe flag) as the VirusTotal score (i.e., 0 means highly malicious and 1 is completely benign). Both WOT and VirusTotal have been used to determine the trustworthiness of a domain by previous studies [29, 31, 38, 39].

One challenge we faced is determining thresholds for trust ratings since it varies widely across different services that report a trustworthiness score. Therefore, to provide a fair analysis, we report the contribution of domains to the ad cost for different thresholds. Figure 16 illustrates the cumulative performance cost of ad domains as a function of trustworthiness assessed by WOT and VirusTotal. For WOT, we use its default classification (5 classes) [31]. For VirusTotal, we observe

Proc. ACM Meas. Anal. Comput. Syst., Vol. 5, No. 1, Article 3. Publication date: March 2021. 


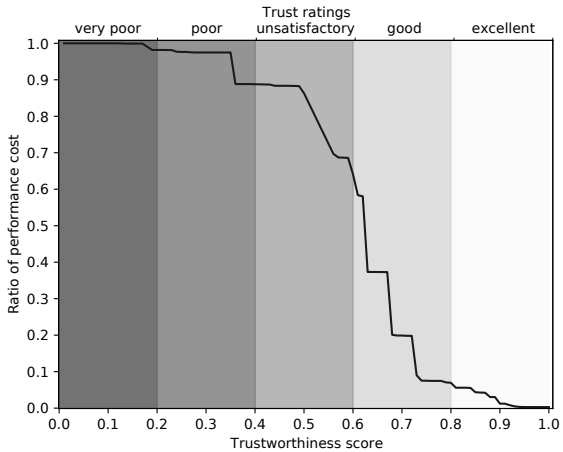

(a) WOT

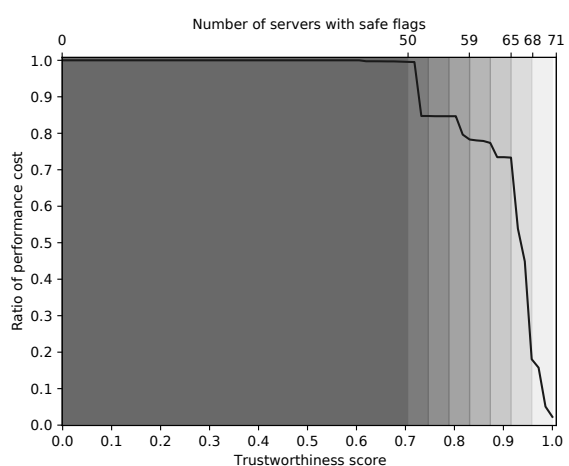

(b) VirusTotal

Fig. 16. Performance cost of ads delivered by ad domains as a function of its trustworthiness score (CDF). Both scores, WOT (top) and VirusTotal (bottom) are normalized to [0,1]. Different colors highlight different trustworthiness rating.

that almost all of the domains receive at least 50 safe flags, so we only breakdown the region from 50 to 71 servers at the granularity of 3 servers.

Finding 12. Following the default classification of WOT, about $63 \%$ of ads cost is from ads delivered by trusted ad domains (excellent and good rating). Nevertheless, domains that are not trusted (unsatisfactory, poor, and very poor rating) contribute to a considerable portion of ads (37\%) which is a flag for publishers. Accordingly, for VirusTotal, we see that only $5 \%$ of the performance cost of ads is from domains that don't receive any red flags.

Finding 13. Domains that are moderately trusted (i.e., neither highly trusted nor untrusted) have the highest contribution to the performance cost of ads as seen from Figure 16. The amount of drop in the fraction of performance cost (y-axis) within each shaded region indicates the performance cost for that level of trust. For example, domains with more than $80 \%$ WOT score (excellent trust rating) contribute to $5 \%$ of ads performance cost while $58 \%$ of ads cost belongs to domains with $60 \%$ to $80 \%$ score (good trust rating). Likewise, domains with less than 3 VirusTotal red flags (first shaded region from the right) account for $18 \%$ of ads cost but $55 \%$ for domains with 3 to 6 red flags (second shaded region from the right). Our results do not assert a strong correlation between trustworthiness and the performance impact of third-party ad domains.

Breakdown of performance cost by popularity. Similar to trustworthiness (gauged by the delivered content), we can study the relationship between the popularity of an ad domain and its performance impact. Accordingly, we first model the domain reputation by its popularity, which is determined by the Alexa ranking [6], and the number of websites in our corpus to which it delivers ads. However, there is no agreed-upon cutoff to split ad domains into popular versus unpopular. For this reason, we follow a similar method to the trustworthiness study and present the performance cost of ad domains at varying cutoff levels. Figure 17 illustrates the cumulative contribution of popular domains to the performance cost of ads for two metrics.

Finding 14. Earlier in this section, we observe no correlation between the popularity of the ad domains (i.e., number of referred websites) and the performance cost for multiple domains. However, at the macro-level, more popular ad domains contribute more to the performance cost as seen from Figure 17(a) and this is due to higher reach of those domains. As highlighted in this figure, the fraction of performance cost drops about $40 \%$ within a $5 \%$ range of the most popular ad domains. However, for the Alexa ranking, we observe multiple sharp drops throughout the 
score range, meaning there exist multiple ad-domains that have a significant contribution to the performance that is neither very popular nor very unpopular.

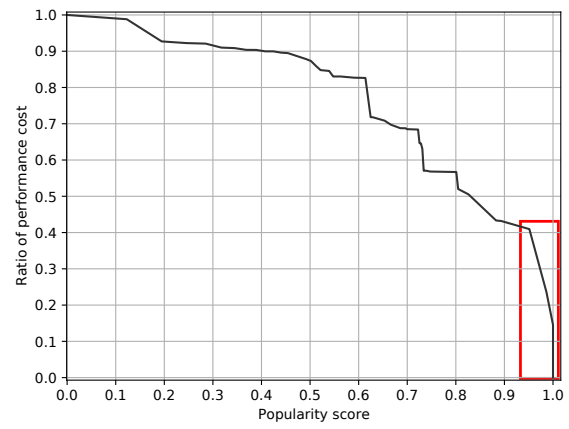

(a) Number of referred websites

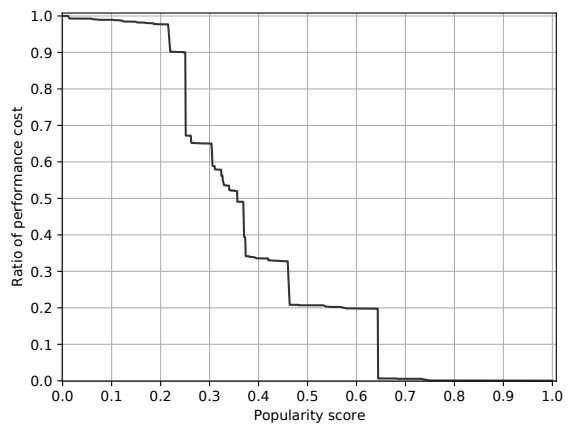

(b) Alexa ranking

Fig. 17. Performance cost of ads from popular domains as a function of popularity score (CDF) on the number of referrers (top) and Alexa ranking (bottom).

\subsection{Desktop vs. Mobile ads}

Mobile represents a significant medium for web consumption. We repeat the experiments and load webpages on the Nexus 6P smartphone to evaluate the performance cost of web ads on mobile devices using adPerf. adPerf uses port forwarding to connect to the device and Chrome remote interface to capture traces remotely on the device which they are then parsed and analyzed on the host system. Figure 18 shows a comparison of the performance cost of web ads on mobile and desktop for the landing page of the News corpus.

Finding 15. Mobile ads add on average $8 \%$ overhead to the page loading computation and the same amount on the network consumption. This is a notable $7 \%$ less than desktop pages. Further breakdown of computation cost by browser activities show a similar contribution of stages to the ads workload, with Scripting being the highest amounting to $87 \%$. However, the amount of scripting work spent on ads to the total scripting workload reduced from $25 \%$ on the laptop to $13 \%$ on mobile.

Our assessment shows that this performance gap is because websites display fewer ads and they are better optimized on mobile to deliver content on a smaller screen. Overall, the fraction of ad documents to the total documents in the news websites dropped from $22.5 \%$ to $15.5 \%$ on the mobile device. Table 4 breaks down the above fraction by each content type $\left(n r_{a d}^{c} / n r_{*}^{c}\right)$ except Media for the mobile version. Across the board, a fewer number of ad documents are fetched in each category compared to results on desktop (Table 3). For instance, ad images and scripts dropped by $44 \%$ and $24 \%$ in the mobile crawl. A recurring pattern in the websites is the absence (or limited inclusion) of skyscraper ads on mobile with a marked difference in the performance cost. This trend is illustrated in Figure 19 for Deutsche Welle, the popular German-based international news broadcaster. In $m . d w . c o m$ (the mobile version on the right), two side skyscraper display ads are replaced with one in-feed ad, reducing $10 \%$ of ads performance overhead.

We investigate the sources of mobile ads and breakdown the performance cost by third-party domains. Table 5 shows the contribution of the top mobile ad domains.

Finding 16. The sources that deliver ad content on mobile are fairly different from the desktop version and they have a dissimilar contribution to the performance cost of web-ads. For example, 


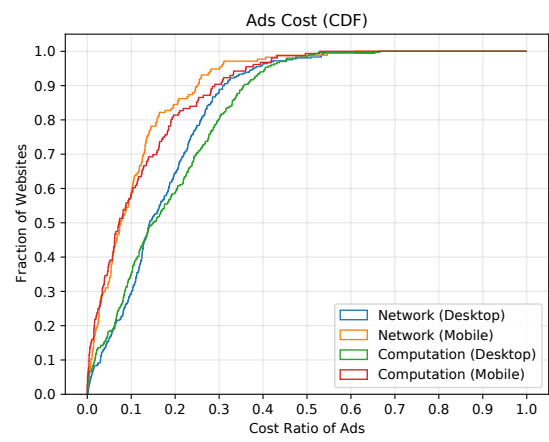

Fig. 18. Comparison of performance cost of ads (computation and network) for mobile and laptop on news corpus.

\begin{tabular}{|c|c|}
\hline cont. type & $n r_{a d}^{c} / n r_{*}^{c}$ \\
\hline Script & $17.5 \%$ \\
\hline HTML & $25.7 \%$ \\
\hline Image & $12.8 \%$ \\
\hline Font & $2.2 \%$ \\
\hline CSS & $2.3 \%$ \\
\hline XML & $36 \%$ \\
\hline XHR & $6.4 \%$ \\
\hline Media & $6.3 \%$ \\
\hline
\end{tabular}

Table 4. Fraction of ad documents to total documents for each content type $\left(n r_{a d}^{c} / n r_{*}^{c}\right)$.

\begin{tabular}{|c|c|}
\hline Domain & Cost \\
\hline doubleclick.net & $28 \%$ \\
\hline googletagservices.com & $20 \%$ \\
\hline googlesyndication.com & $18 \%$ \\
\hline ampproject.com & $7 \%$ \\
\hline cloudfront.net & $4 \%$ \\
\hline 2mdn.net & $4 \%$ \\
\hline
\end{tabular}

Table 5. Top ad domains contribution to performance cost of mobile ads.

about $7 \%$ of the performance of ads on mobile comes from ampproject.com that specifically provides optimized ads for AMP (Accelerated Mobile Pages). Similar to desktop, doubleclick. net and googletagservices. com have the highest contribution in mobile advertising with a combined $48 \%$ share of performance cost, $13 \%$ more compared to desktop.

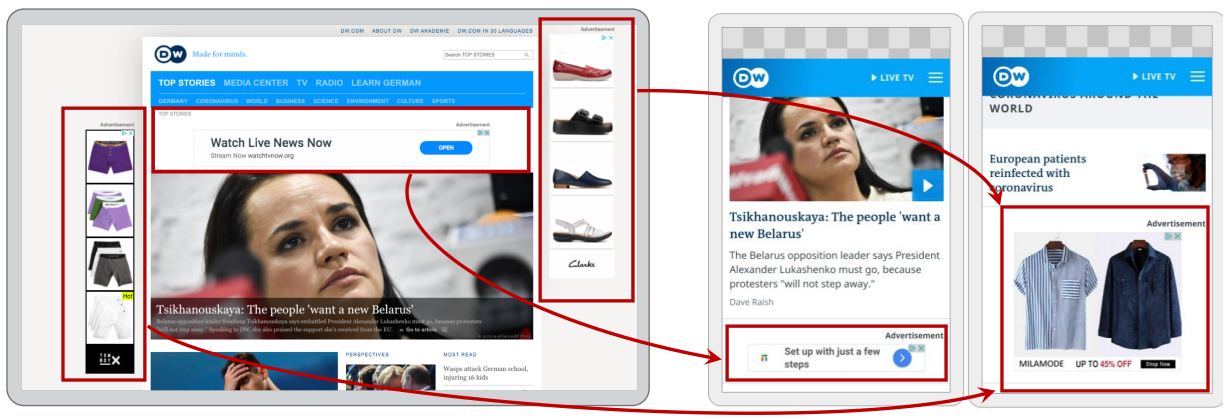

Fig. 19. Snapshot of Deutsche Welle website on laptop (left) and mobile (right). Two side skyscrapper ads are substituted with one in-feed ad on the mobile.

\subsection{Applications}

AdPerf and the measurement study from different viewpoints present multiple applications for web researchers, browser developers, ad designers, content publishers, and perhaps even users. Notwithstanding, we discuss two use-cases below.

Heavy-ad intervention. Ads that consume a disproportionate amount of resources such as draining the battery or eating up bandwidth on a device negatively impact the user experience. Intrusive ads range from the actively malicious, such as crypto-miners, to benign content with inadvertent bugs or performance issues. Chrome is launching an extension to limit the resources an ad may use and unloading that ad if the limits are exceeded. Tentatively, they define the following criteria and coarse-grained thresholds to limit the ads [15].

- Uses the main thread (i.e., the renderer thread which executes the majority of the computation activities) for more than 60 seconds in total or more than 15 seconds in any 30-second window. 
- Uses more than 4 megabytes of network bandwidth.

The above metrics have been reported to have blocked many non-intrusive ads by Google Ad Manager Native Video and YouTube Skippable Preroll ads [10, 16]. AdPerf can aid Google engineers and potentially other browser developers to extensively study and characterize the performance of intrusive heavy-ads in different computation stages and network resources to establish a fine-grained threshold and criteria.

High-performance ads. AdPerf provides insight and guidance to both publishers and thirdparty ad providers to improve the performance of ads by identifying the stage and/or resource which are the main bottlenecks. For example, if adPerf identifies Scripting to be the computation and network bottleneck of ads on a website, one can follow targeted optimizations to loading third-party JavaScript such as lazy-loading scripts and libraries (e.g., serving an ad in the footer only when a user scrolls down the page), splitting JavaScript bundles (e.g., dynamic import() statement), self-hosting scripts with Service workers particularly for ad domains with consistent APIs, using resource hints like preconnect and DNS-prefetch, sandboxing script with iframes, using asynchronous ad tag manager in the code, and other cognate recommendations provided by Google Page Insights [18] and Lighthouse [17]. Likewise, if Painting turns out to have excessive computation overhead for ads, this is likely due to animated GIF in the background of ad iframe or animation triggered by CSS (e.g., @keyframe rules). Ad designers can follow the recommendations on painting optimization, such as limiting manipulation to transform and opacity CSS properties that avoid repainting. Our analysis on ad domains can also advise publishers to select their ad providers from reliable syndications (i.e., with satisfactory trustworthiness score) that at the same time have a minimal performance impact, considering their reach.

\section{RELATED WORK}

Over the past years, there have been a handful of studies on the performance characterization of web browsers and online advertising. Prior research mainly uses adblocker and adheres to page load time (PLT) as the performance metric for characterizing the computation cost of ads; i.e., compare PLT before and after content blocking to measure ads cost. On the other hand, adPerf uses the ratio of the ad workload to the main content workload. Although adPerf's metric provides additional insight into the computation cost of ads, it cannot be directly compared to PLT for two reasons: (i) PLT combines both network and computation cost into a single metric and (ii) parallelization among activities in the browser. Moreover, with adblocking, we cannot decompose the performance cost into lower-level browser stages (e.g., HTML parsing and JavaScript) since they block resources at network initiation, and subsequent resource parsing, evaluation, and rendering are not captured. Therefore, in this section, we quantitatively and/or illustratively compare related work against adPerf.

\subsection{Performance analysis of ads}

Garimella et al. [36] analyze the performance efficiency and network overhead of popular ad blockers such as Adblock Plus [4], Ghostery [13], uBlock [22], etc. According to their data, blocking ads with Adblock Plus (Easylist rules) saves roughly $34 \%$ on cumulative network request time. This is higher than our measured network cost (15\%) without the deployment of an adblocker. Although their setup is different and their corpus of news websites is small (Alexa top 150), they observe an increase in the number of network requests. This is due to various tracking services of their own and request for JavaScript modules designed for counter ad-blocking. Besides, they report a $15 \%$ to $43 \%$ increase in the CPU wall-clock when they use ad-blockers (Adblock, Adblock Plus, ublock, 
and Privacy Badger) and conclude that the time to load pages is not necessarily faster due to the overhead of ad blockers.

Butkiewicz et al. [30] break down the content of non-origin requests by MIME type and reports images and HTML/XML contribute to $42 \%$ and $9 \%$ respectively, which is slightly higher than our measurements, whereas, JavaScript contribution (25\%) is far less than our measurements. Given the fact that $70 \%$ of these non-origin requests belong to advertising and analytics, this comparison signifies the rise of responsive and interactive ads within the past few years. Additionally, they attempt to quantify the cost of third-party content on page load time. By blocking non-origin content (using custom adblock filter), they measure 25\% contribution. However, they report a $15 \%$ contribution when they consider the impact of non-origin requests on wall-clock rather than content blocking. Although their latter method does not preclude the content blocking overhead, it dismisses the parallelization among network requests and browser rendering activities associated with resources, hence is not reliable.

In other related studies $[50,56]$, authors deploy ad blockers in the wild and then use passive measurements on the traces to characterize the network traffic. Both studies report $17-18 \%$ of the network requests to belong to adverts, which is close to our numbers. Similar to our measurements, in [50], network requests are broken down by content type. However, the authors do not completely isolate the content types (e.g., CSS and JavaScript are not categorized), and therefore, a direct comparison is not feasible. Nevertheless, none of the studies investigate the effect of ads on the computation cost of page loading.

\subsection{Performance analysis of browsers}

Another notable line of research concerns the performance analysis of browsers given their complexity, large codebase, and multi-process execution. The majority of browser vendors have an integrated profiler. Examples include the Chrome profiler [21] for Google Chrome and Gecko profiler [12] for Mozilla Firefox, which provides statistics about task timing, call graph, memory usage, and network activities. There have also been several efforts on critical path analysis [37, 46, 54, 55]. Wprof extracts the dependency graph and breaks down the activities based on type (computation and network activities) $[46,54]$. Coz+ [48] generates quantitative what-if graphs about the dynamic behavior of the critical path, based on the idea of causal profiling [32, 49]. By analyzing Alexa top webpages, the above study concludes that JavaScript is the critical computation activity, and $40 \%$ improvement of this stage improves page loading performance by $8.5 \%$. Our approach to performance analysis is similar in spirit to the above studies. However, prior efforts did not distinguish between the time spent in the different browser stages/activities based on the resource (ads vs. main content).

\section{CONCLUSIONS AND TAKEAWAYS}

Our evaluations on the performance cost of ads lead to multiple new and interesting observations. The key finding of this research is that ads have a significant cost, more than $15 \%$ of the computation workload. This cost is relatively less in mobile browsing due to fewer and optimized ads for a smaller screen. Moreover, we discover Scripting contributes to $\approx 88 \%$ of this cost in both environments suggesting ad designers to focus more on optimizing their JavaScript codes and publishers to follow practices for lazy loading of these scripts. We also find that ads have a different fingerprint on browser activities and web documents compared to the main content. For example, HTML parsing takes up only $5 \%$ of browser page loading workload (the lowest among other stages) but $29 \%$ of that is spent on ad-related content, more than any other stage, and XML files are requested more by the ad contents compared to the primary contents. Practitioners can use this anomaly to build a system for detection and intervention of ads or a subset of them (e.g., intrusive ads). Our evaluation 
also shows that a considerable fraction of the performance cost of ads is from untrusted domains which is a signal for the web community and to publishers to reconsider their ad-delivery network.

In this study, we did not account for ad resources that might be directly embedded in native HTML and ad resources that cannot be detected by filter lists (i.e., websites that use circumvention to evade filter lists). In future work, we plan to also include such sources of ad content. This addition would only increase the performance costs of different ad breakdowns reported throughout this paper, which we believe is already significant enough to warrant deeper attention. This work primarily aimed at designing a methodology and open-source infrastructure for fine-grained analysis of ads which we anticipate to be a useful tool for web researchers to prioritize their optimization efforts on web ads and publishers to analyze the impact of ads on their websites.

\section{ACKNOWLEDGEMENTS}

We thank the anonymous reviewers and our shepherd, Arif Merchant from Google, for his careful reading and constructive feedback. This project is partially supported by the National Science Foundation (NSF) under award number 1939237.

\section{REFERENCES}

[1] 2020. AD BLOCK DETECTION SCRIPT. https://iabtechlab.com/software/ad-block-detection-script/.

[2] 2020. Ad blocking user penetration rate in the United States. https://www.statista.com/statistics/804008/ad-blockingreach-usage-us.

[3] 2020. AdBlock. https://getadblock.com/.

[4] 2020. Adblock Plus. https://adblockplus.org.

[5] 2020. adblockparser. https://github.com/scrapinghub/adblockparser.

[6] 2020. Alexa Top News Sites. https://www.alexa.com/topsites/category/News.

[7] 2020. Alexa Top Sites. https://www.alexa.com/topsites/countries/US.

[8] 2020. Chrome DevTools Protocol. https://chromedevtools.github.io/devtools-protocol.

[9] 2020. Chrome Devtools Timeline. https://developers.google.com/web/tools/chrome-devtools/evaluate-performance/ timeline-tool.

[10] 2020. Chrome's coming changes to video ad blocking could impact YouTube. https://martechtoday.com/chromescoming-changes-to-video-ad-blocking-could-impact-youtube- 238360.

[11] 2020. EasyList. https://easylist.to.

[12] 2020. Gecko profiler. https://developer.mozilla.org/en-US/docs/Mozilla/Performance/Profiling_with_the_Builtin_Profiler.

[13] 2020. Ghostery. https://www.ghostery.com/.

[14] 2020. Global internet advertising revenue in 2015 and 2020. https://www.statista.com/statistics/237800/global-internetadvertising-revenue/.

[15] 2020. Handling Heavy Ad Interventions. https://developers.google.com/web/updates/2020/05/heavy-ad-interventions.

[16] 2020. Heavy Ads: (brief description of issue). https://bugs.chromium.org/p/chromium/issues/detail?id=1114329.

[17] 2020. Lighthouse. https://developers.google.com/web/tools/lighthouse.

[18] 2020. Loading Third-Party JavaScript. https://developers.google.com/web/fundamentals/performance/optimizingcontent-efficiency/loading-third-party-javascript/?utm_source=lighthouse\&utm_medium=unknown.

[19] 2020. Number of active desktop adblock plugin users worldwide. https://www.statista.com/statistics/435252/adblockusers-worldwide/.

[20] 2020. Popeteer. https://developers.google.com/web/tools/puppeteer/get-started.

[21] 2020. The Trace Event Profiling Tool. https://www.chromium.org/developers/how-tos/trace-event-profiling-tool.

[22] 2020. uBlock. https://ublock.org/.

[23] 2020. VirusTotal. https://www.virustotal.com.

[24] 2020. Website Safety, Security Check Web Of Trust. https://www.mywot.com/.

[25] 2020. Zbrowse. https://github.com/zmap/zbrowse.

[26] Daniel An. 2018. Mobile page speed. https:/www.thinkwithgoogle.com/marketing-resources/data-measurement/ mobile-page-speed-new-industry-benchmarks/.

[27] Gary Anthes. 2014. Data brokers are watching you. Commun. ACM 58, 1 (2014), 28-30.

[28] Muhammad Ahmad Bashir, Sajjad Arshad, William Robertson, and Christo Wilson. 2016. Tracing information flows between ad exchanges using retargeted ads. In 25th USENIX Security Symposium. 481-496.

Proc. ACM Meas. Anal. Comput. Syst., Vol. 5, No. 1, Article 3. Publication date: March 2021. 
[29] Hamad Binsalleeh. 2014. Analysis of Malware and Domain Name System Traffic. Ph.D. Dissertation. Concordia University.

[30] Michael Butkiewicz, Harsha V Madhyastha, and Vyas Sekar. 2011. Understanding website complexity: measurements, metrics, and implications. In Proceedings of the 2011 ACM SIGCOMM conference on Internet measurement conference. 313-328.

[31] Pern Hui Chia and Svein Johan Knapskog. 2011. Re-evaluating the wisdom of crowds in assessing web security. In International Conference on Financial Cryptography and Data Security. Springer, 299-314.

[32] Charlie Curtsinger and Emery D Berger. 2015. COZ: Finding Code that Counts with Causal Profiling. In Proceedings of the 25th Symposium on Operating Systems Principles. ACM, 184-197.

[33] Alexandre De Corniere and Romain De Nijs. 2016. Online advertising and privacy. The RAND fournal of Economics 47, 1 (2016), 48-72.

[34] Steven Englehardt and Arvind Narayanan. 2016. Online tracking: A 1-million-site measurement and analysis. In Proceedings of the 2016 ACM SIGSAC conference on computer and communications security. ACM, 1388-1401.

[35] David S Evans. 2009. The online advertising industry: Economics, evolution, and privacy. fournal of economic perspectives 23, 3 (2009), 37-60.

[36] Kiran Garimella, Orestis Kostakis, and Michael Mathioudakis. 2017. Ad-blocking: A study on performance, privacy and counter-measures. In Proceedings of the 2017 ACM on Web Science Conference. ACM, 259-262.

[37] Hossein Golestani, Scott Mahlke, and Satish Narayanasamy. 2019. Characterization of Unnecessary Computations in Web Applications. In 2019 IEEE International Symposium on Performance Analysis of Systems and Software (ISPASS) IEEE, 11-21.

[38] Muhammad Ikram and Mohamed Ali Kaafar. 2017. A first look at mobile ad-blocking apps. In 2017 IEEE 16th International Symposium on Network Computing and Applications (NCA). IEEE, 1-8.

[39] Muhammad Ikram, Rahat Masood, Gareth Tyson, Mohamed Ali Kaafar, Noha Loizon, and Roya Ensafi. 2019. The chain of implicit trust: An analysis of the web third-party resources loading. In The World Wide Web Conference. ACM, 2851-2857.

[40] Umar Iqbal, Zubair Shafiq, and Zhiyun Qian. 2017. The ad wars: retrospective measurement and analysis of anti-adblock filter lists. In Proceedings of the 2017 Internet Measurement Conference. ACM, 171-183.

[41] Umar Iqbal, Zubair Shafiq, Peter Snyder, Shitong Zhu, Zhiyun Qian, and Benjamin Livshits. 2018. Adgraph: A machine learning approach to automatic and effective adblocking. arXiv preprint arXiv:1805.09155 (2018).

[42] Adam Lerner, Anna Kornfeld Simpson, Tadayoshi Kohno, and Franziska Roesner. 2016. Internet jones and the raiders of the lost trackers: An archaeological study of web tracking from 1996 to 2016. In 25th USENIX Security Symposium.

[43] Zhou Li, Kehuan Zhang, Yinglian Xie, Fang Yu, and XiaoFeng Wang. 2012. Knowing your enemy: understanding and detecting malicious web advertising. In Proceedings of the 2012 ACM conference on Computer and communications security. ACM, 674-686.

[44] Leo A Meyerovich and Rastislav Bodik. 2010. Fast and parallel webpage layout. In Proceedings of the 19th international conference on World wide web. ACM, 711-720.

[45] Muhammad Haris Mughees, Zhiyun Qian, and Zubair Shafiq. 2017. Detecting anti ad-blockers in the wild. Proceedings on Privacy Enhancing Technologies 2017, 3 (2017), 130-146.

[46] Javad Nejati and Aruna Balasubramanian. 2016. An In-depth study of Mobile Browser Performance. In Proceedings of the 25th International Conference on World Wide Web. 1305-1315.

[47] Rishab Nithyanand, Sheharbano Khattak, Mobin Javed, Narseo Vallina-Rodriguez, Marjan Falahrastegar, Julia E Powles, Emiliano De Cristofaro, Hamed Haddadi, and Steven J Murdoch. 2016. Adblocking and counter blocking: A slice of the arms race. In 6th USENIX Workshop on Free and Open Communications on the Internet (FOCI 16).

[48] Behnam Pourghassemi, Ardalan Amiri Sani, and Aparna Chandramowlishwaran. 2019. What-If Analysis of Page Load Time in Web Browsers Using Causal Profiling. Proceedings of the ACM on Measurement and Analysis of Computing Systems 3, 2 (2019), 1-23.

[49] Behnam Pourghassemi, Ardalan Amiri Sani, and Aparna Chandramowlishwaran. 2021. Only Relative Speed Matters: Virtual Causal Profiling. ACM SIGMETRICS Performance Evaluation Review (2021).

[50] Enric Pujol, Oliver Hohlfeld, and Anja Feldmann. 2015. Annoyed users: Ads and ad-block usage in the wild. In Proceedings of the 2015 Internet Measurement Conference. ACM, 93-106.

[51] M Zubair Rafique, Tom Van Goethem, Wouter Joosen, Christophe Huygens, and Nick Nikiforakis. 2016. It's free for a reason: Exploring the ecosystem of free live streaming services. In Proceedings of the 23rd Network and Distributed System Security Symposium (NDSS 2016). Internet Society, 1-15.

[52] RJG Simons and Aiko Pras. 2010. The hidden energy cost of web advertising. In Proceedings of the 12th Twente Student Conference on Information Technology. 1-8.

[53] Peter Snyder, Antoine Vastel, and Ben Livshits. 2020. Who Filters the Filters: Understanding the Growth, Usefulness and Efficiency of Crowdsourced Ad Blocking. Proceedings of the ACM on Measurement and Analysis of Computing 
Systems 4, 2 (2020), 1-24.

[54] Xiao Sophia Wang, Aruna Balasubramanian, Arvind Krishnamurthy, and David Wetherall. 2013. Demystifying Page Load Performance with WProf.. In 10th USENIX Symposium on Networked Systems Design and Implementation (NSDI 13). 473-485.

[55] Zhen Wang, Felix Xiaozhu Lin, Lin Zhong, and Mansoor Chishtie. 2011. Why Are Web Browsers Slow on Smartphones? In Proceedings of the 12th Workshop on Mobile Computing Systems and Applications. 91-96.

[56] Craig E Wills and Doruk C Uzunoglu. 2016. What ad blockers are (and are not) doing. In 2016 Fourth IEEE Workshop on Hot Topics in Web Systems and Technologies (HotWeb). IEEE, 72-77.

[57] Zhonghao Yu, Sam Macbeth, Konark Modi, and Josep M Pujol. 2016. Tracking the trackers. In Proceedings of the 25th International Conference on World Wide Web. 121-132.

[58] Shitong Zhu, Umar Iqbal, Zhongjie Wang, Zhiyun Qian, Zubair Shafiq, and Weiteng Chen. 2019. ShadowBlock: A Lightweight and Stealthy Adblocking Browser. In The World Wide Web Conference. ACM, 2483-2493.

Received October 2020; revised December 2020; accepted January 2021 